\title{
A review of communication-oriented optical wireless systems
}

\author{
Deva K Borah ${ }^{1}$, Anthony C Boucouvalas ${ }^{2}$, Christopher C Davis ${ }^{3}$, Steve Hranilovic ${ }^{4}$ and Konstantinos Yiannopoulos ${ }^{5^{*}}$
}

\begin{abstract}
This article presents an overview of optical wireless (OW) communication systems that operate both in the short(personal and indoor systems) and the long-range (outdoor and hybrid) regimes. Each of these areas is discussed in terms of (a) key requirements, (b) their application framework, (c) major impairments and applicable mitigation techniques, and (d) current and/or future trends. Personal communication systems are discussed within the context of point-to-point ultra-high speed data transfer. The most relevant application framework and related standards are presented, including the next generation Giga-IR standard that extends personal communication speeds to over 1 $\mathrm{Gb} / \mathrm{s}$. As far as indoor systems are concerned, emphasis is given on modeling the dispersive nature of indoor OW channels, on the limitations that dispersion imposes on user mobility and dispersion mitigation techniques. Visible light communication systems, which provide both illumination and communication over visible or hybrid visible/ infrared LEDs, are presented as the most important representative of future indoor OW systems. The discussion on outdoor systems focuses on the impact of atmospheric effects on the optical channel and associated mitigation techniques that extend the realizable link lengths and transfer rates. Currently, outdoor OW is commercially available at $10 \mathrm{~Gb} / \mathrm{s}$ Ethernet speeds for Metro networks and Local-Area-Network interconnections and speeds are expected to increase as faster and more reliable optical components become available. This article concludes with hybrid optical wireless/radio-frequency (OW/RF) systems that employ an additional RF link to improve the overall system reliability. Emphasis is given on cooperation techniques between the reliable RF subsystem and the broadband OW system.
\end{abstract}

Keywords: optical wireless, personal communication systems, indoor systems, outdoor systems, hybrid OW/RF systems

\section{Introduction}

It has been more than 30 years since optical wireless (OW) was proposed as an alternative broadband technology for wireless data transmission applications [1]. The underlying concept of OW is very simple: utilize optical beams to carry data through the atmosphere or vacuum. As a result, OW link architectures are very similar to optical fiber communication point-to-point links, with the exception that no optical fibers are deployed as a transmission medium. OW is also very similar to RF wireless, but radio waves are replaced with light and antennas with free-space optical transceivers. Despite this superficial resemblance between OW and

\footnotetext{
* Correspondence: kyianno@uop.gr

${ }^{5}$ Department of Telecommunications Science and Technology, University of Peloponnese, Tripoli, Greece

Full list of author information is available at the end of the article
}

RF links, OW exhibits several appealing attributes when compared to RF. OW links are inherently broadband and optical frequencies in the infrared and visible spectrum are neither regulated nor licensed. Optical components are also cheaper and consume less electrical power than high-speed RF components. Finally, OW links do not suffer from multipath fading and have much less potential for interference with RF-sensitive electronic systems. These advantages do not, however, imply that OW is a universal replacement for RF communications. The application of OW systems is limited when considering area coverage and user mobility, where RF technologies prove invaluable. In addition, OW systems operate under strict eye safety regulations, while at the same time incoherent OW receivers present lower sensitivity than their RF counterparts because of their photo-electric conversion mechanisms and the 
Table 1 Comparison between RF and OW systems

\begin{tabular}{|c|c|c|}
\hline & OW & $\mathrm{RF}$ \\
\hline Bandwidth & Not regulated & Licensed \\
\hline Available line rates & $<10 \mathrm{~Gb} / \mathrm{s}$ & $<1.25 \mathrm{~Gb} / \mathrm{s}$ \\
\hline Path losses & High & High \\
\hline Multipath fading & $\begin{array}{l}\text { No } \\
\text { (large collector } \\
\text { area) }\end{array}$ & Yes \\
\hline $\begin{array}{l}\text { Multipath } \\
\text { distortion }\end{array}$ & $\begin{array}{l}\text { Only in diffuse } \\
\text { indoor systems }\end{array}$ & Yes \\
\hline Noise sources & Ambient light & $\begin{array}{l}\text { Interference from other users, } \\
\text { electrical noise }\end{array}$ \\
\hline Detection type & Incoherent & Coherent/Incoherent \\
\hline SNR & $\begin{array}{l}\text { Depends on } \\
\text { optical signal } \\
\text { power }\end{array}$ & $\begin{array}{l}\text { Depends on RF signal } \\
\text { amplitude }\end{array}$ \\
\hline Receiver sensitivity & Low & High \\
\hline Eye safety & Required & N/A \\
\hline $\begin{array}{l}\text { Electromagnetic } \\
\text { compatibility }\end{array}$ & Yes & Conditional \\
\hline
\end{tabular}

impact of ambient light noise sources. Table 1 summarizes the main differences between OW and RF systems.

To better understand the place of OW systems in the wireless world, Figure 1 summarizes state-of-the art commercial RF and OW technologies, as well as technologies under standardization by major bodies including IEEE, 3GPP, Bluetooth and IrDA. Technologies are presented with respect to their area of coverage, ranging from a few centimeters in personal communications to over $1 \mathrm{~km}$ in outdoor communications, and the data rates they attain, including low rate legacy links under $1 \mathrm{Mb} / \mathrm{s}$ (Bluetooth and older IrDA systems). Clearly, contemporary OW links provide channel rates up to $10 \mathrm{~Gb} / \mathrm{s}$, which directly compare to the ones of optical fibers. At the same time, commercial OW links operate at link distances that are challenging to attain in RF (3G/4G) and millimeter-wave (60 GHz) broadband communications.

$\mathrm{OW}$ is a unique technology that provides an attractive alternative in niche application areas, complementing fiber-optic and RF wireless solutions when they are either too costly to deploy, create undesirable interference, or are not feasible at all. Figure 2 illustrates some of the application areas in which OW has been successfully applied. Two mainstream application areas of OW are last-mile broadband access and office interconnection; both are the business objectives of a number of component and system manufacturers. In such applications, state-of-the-art OW systems support $10 \mathrm{~Gb} / \mathrm{s}$ Ethernet, which equals the bandwidth provided by metro fiber optic systems and is significantly higher than the $1.25 \mathrm{~Gb} / \mathrm{s}$ Ethernet provided by competing RF wireless systems that operate in the $60 \mathrm{GHz}$ frequency range. At the same time the deployment cost of OW systems is significantly lower than that of fiber optics, which can easily reach $\$ 1 \mathrm{M} / \mathrm{mile}$ in urban areas. OW installation only requires the alignment of two freespace optical transceivers rather than digging trenches and repairing roads.

Another major application area of OW is in personal communication systems. The rapid progress in optical technology over the past 30 years, driven mainly by fiber-optic systems and display technologies, has enabled the mass production of high volume, low cost, and fast optical components that are suitable for short range OW. During the 1990s OW emerged as a candidate technology for data transmission in personal communications systems using protocols developed by the Infrared Data Association [2]. The result of this study was the standardization and commercialization of OW ports

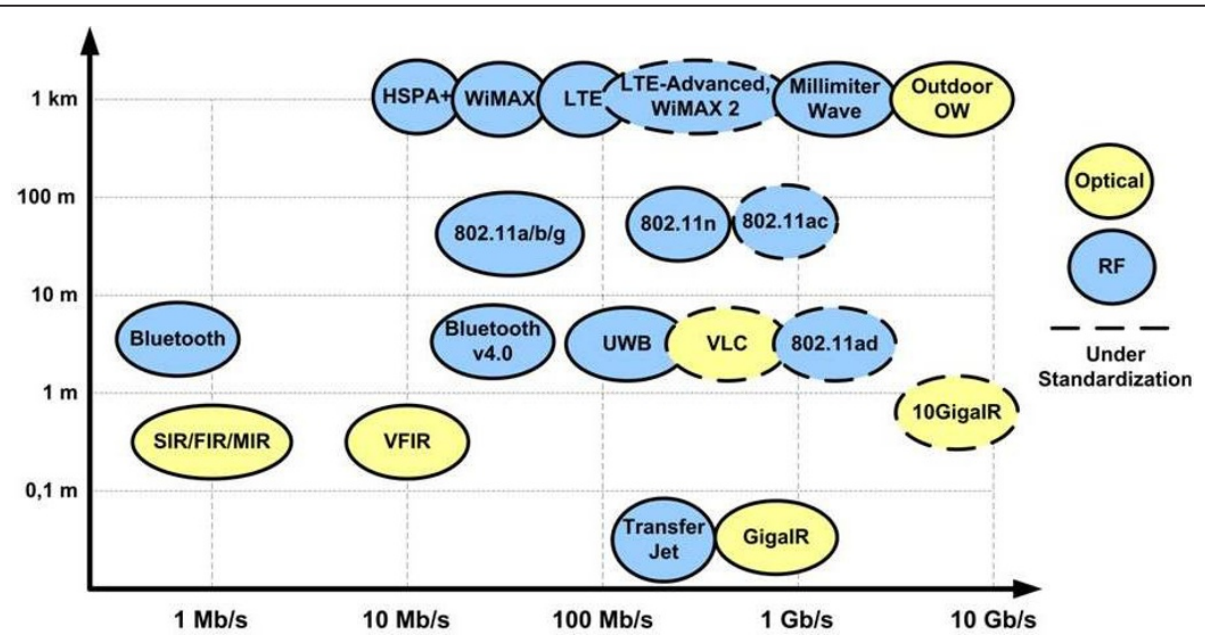

Figure $1 \mathrm{RF}$ and $\mathrm{OW}$ technologies as defined in standards and deployed in commercial products. 


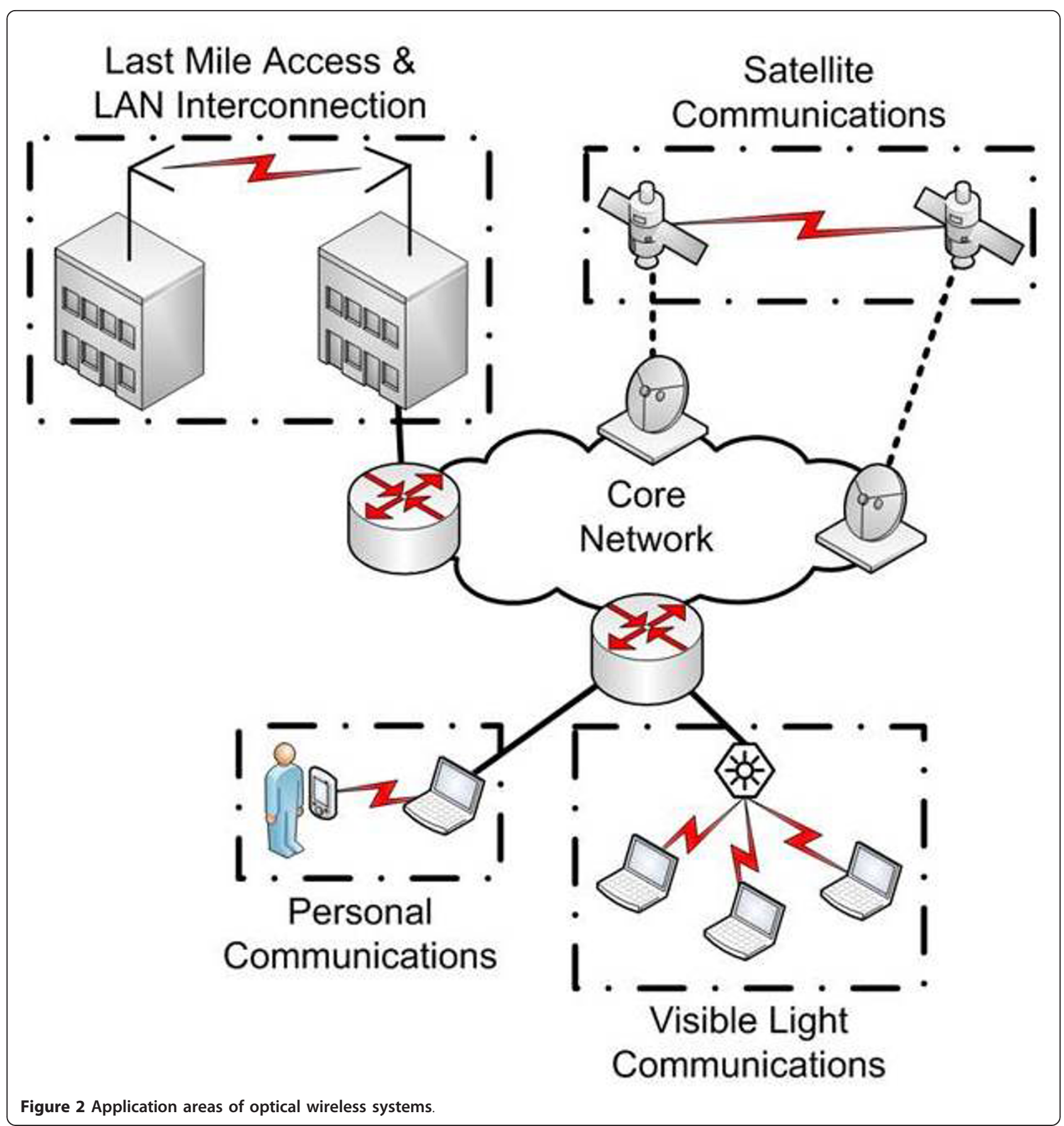

that have been extremely popular since the late 1990s and may be found on all kinds of mobile phones and portable computers. The current state-of-the-art in personal communications is Gigabit Infrared (Giga-IR) that operates at link speeds of $512 \mathrm{Mb} / \mathrm{s}$ and $1.024 \mathrm{~Gb} / \mathrm{s}$. This short-range interconnection is among the highest speed wireless interconnection media available and is easily integrated into portable and consumer devices. At the Expo Comm Wireless Japan in 2008 and 2009,
KDDI Corporation demonstrated Giga-IR, which is envisioned as an interconnection medium for future cellular phones.

Figure 2 also illustrates two additional application areas of OW: indoor and ultra-long-haul systems. OW was initially proposed as a broadband transmission technology for short range diffuse indoor wireless systems [1]. Indoor OW systems are currently being revisited within the framework of Visible Light Communication 
(VLC) Systems [3] that aim to combine lighting and communications over commercially available visible light LEDs. With respect to ultra-long-haul systems, major technological advances in the fields of adaptive optics and beam acquisition/tracking, which are functions of critical importance in ultra-long-haul outdoor OW links, have enabled the deployment of tractable broadband outdoor OW communication systems. At the time of writing of this article, numerous ultra-long-haul outdoor OW systems have been proposed and demonstrated in a wide range of applications, including terrestrial, earth-to-satellite, earth-to-high altitude platform, intersatellite, and interplanetary communication links [4].

OW is a standalone technology, differentiated from fiber optics and RF wireless as far as usage scenarios are concerned. OW fits perfectly well into the broad scenario of telecommunication networks as a complementary technology that provides communication solutions tailored to diverse application areas. This article aims to present progress in OW systems, underlying principles that lay the basis of OW, and some of the newest trends that appear to be of practical importance in the future. To facilitate the presentation, OW systems have been categorized in four distinct areas: personal communication systems, indoor communication systems, outdoor communication systems, and hybrid OW/RF systems. Personal communication systems are presented in Section 2. The discussion focuses on the most relevant user requirements and standards (IrDA and IEEE), and also includes the Giga-IR extensions that are the latest trend in short range OW systems. Section 3 presents indoor communication systems, with emphasis given to suitable channel models for the diffuse OW link and techniques for achieving spatial coverage. Section 3 also details the latest trend in indoor OW, Visible-Light Communication Systems. Outdoor OW systems are discussed in Section 4. Emphasis is given to the negative impact that the atmosphere has on optical beams, as well as suitable mitigation techniques that largely alleviate this impact. The latest advances in the most challenging application area of outdoor OW systems, satellite, and high-altitude-platform communications, are also presented. This article concludes with Section 5, which presents outdoor hybrid OW/RF systems. The discussion revolves around the cooperation schemes between the OW and RF links that achieve increased system reliability and capacity availability.

\section{Personal communication systems}

Short-range OW has attracted considerable attention for personal communication systems over the past decade, owing to the fact that OW systems offer a viable, low cost and complexity, high bandwidth solution to user- terminal connectivity. OW provides considerable unlicensed (free) bandwidth in the infrared spectrum, the use of which results in significant cost savings to equipment manufacturers since they do not have to pay an extra fee for using this spectrum and their transceiver designs do not have to strictly conform to spectral masks. Additionally, OW is preferable when health, safety, and aesthetics issues are raised to challenge personal communication systems, since its low transmission powers, short link distances, low profile transceivers and limited spatial coverage make OW one of the least obtrusive personal network technologies. Finally, OW neither suffers from nor directly creates RF interference and OW systems can operate side-by-side with RF systems in personal communication networks. The highspeed electronics that drives OW transmissions must be designed not to be a indirect source of RF interference.

Apart from the generic requirements mentioned above, OW has been quite successful in personal communication systems since it exhibits attributes that closely match key requirements in these systems and their applications [2]. From an application perspective, applications like contact information exchange or file transfer between mobile devices are required to have a very short interaction time. In addition, several applications, for instance secure payment, require increased security and immunity to interference. The short distances involved and the limited spatial coverage of OW, which results from the narrow beams that are utilized, provide inherent security, and interference rejection, while at the same time allow for faster completion of the device discovery process that affects the application interaction time. Moreover, the abundant bandwidth of OW transceivers, more than $1 \mathrm{~Gb} / \mathrm{s}$ at the time of writing of this article, facilitates speedy file transmissions and as a net result the total interaction time is minimized.

The suitability of OW for personal communication systems has drawn the attention of two key players in personal communication systems: IEEE and the Infrared Data Association (IrDA). IEEE had included an OW option in its original 802.11 MAC and PHY standards [5]. The standards defined a wireless communication scheme using diffuse optical channels at 1 and $2 \mathrm{Mb} / \mathrm{s}$. However, they were never updated and are now rendered obsolete. Recently, IEEE launched a separate Task-Group within the framework of 802.15 (IEEE 802.15.7), which is responsible for producing MAC and PHY standards for OW communications based on visible light [6]. IEEE activities will be further discussed in the next section that details indoor systems.

The IrDA aims at equipping personal communication devices (computers, cellular phones, PDAs, etc.) with low-cost and high-bandwidth OW equipment. To this end, IrDA has produced numerous standards for the 
definition and implementation of (a) an infrared physical layer, (b) a protocol stack that is well suited for shortrange OW, and (c) several application scenarios. Even though IrDA had produced standards for multipleaccess implementations over infrared links (Advanced Infrared-AIr) [7-9], with possible applications in personal and local area networks (PANs and LANs), it soon became evident that it will be quite challenging for $\mathrm{OW}$ to compete with RF in terms of area coverage and user mobility. As a result, IrDA has focused primarily on point-and-shoot usage scenarios, since OW is well suited for this [10]: point-and-shoot applications require ultra-fast connection establishment and data transfer; both are readily offered by OW. The rest of the section focuses on the IrDA protocol stack, compliant applications and a comprehensive description of related research efforts.

\subsection{The IrDA protocol stack and related applications}

The IrDA protocol stack is shown in Figure 3. The lower layer is the physical layer [11,12] (IrPHY), which operates at a nominal wavelength of $880 \mathrm{~nm}$, although in practice infrared transceivers are allowed to operate in a range that extends from 850 to $900 \mathrm{~nm}$. LEDs and silicon PIN photodiodes are utilized to reduce the system cost to an absolute minimum at lower speeds, while lasers are a more costly alternative at higher speeds. The average optical powers transmitted by lasers and LEDs are limited by eye and skin safety standards, according to [13]. Transmission (line) speeds vary from a couple of $\mathrm{kb} / \mathrm{s}$ to $1 \mathrm{~Gb} / \mathrm{s}$ and IrDA has provided detailed physical layer specifications for the following communication speeds:

- Serial Infrared (SIR): SIR is 16550 UART compatible, providing link speeds at 2.4, 9.6, 19.2, 38.4, 57.6, and $115.2 \mathrm{~kb} / \mathrm{s}$. The bytes that are to be transmitted over the infrared link are first placed in a 16550 UART chip, which is used to modulate the driving current at the transmitter LED. The UART chip appends a start and a stop bit to the transmitted byte, inverts the bit pattern and also alters the duty cycle of the ' 1 's in the transmitted 10-bit UART frame to 3/16.

- Medium Infrared (MIR): MIR speeds are 0.576 and $1.152 \mathrm{Mb} / \mathrm{s}$. Line coding is almost identical to the SIR speeds, with the only exception being the duty cycle of the '1's in MIR speeds (1/4 instead of 3/16).

- Fast Infrared (FIR): FIR supports a single line speed of $4 \mathrm{Mb} / \mathrm{s}$. Line coding in FIR speeds follows a pulseposition-modulation (PPM) scheme with two-bit symbols. Four chips are required to transmit all possible symbols, and the symbol to chip mapping is defined as $\{00,1000\},\{01,0100\},\{10,0010\}$, and $\{11,0001\}$.

- Very Fast Infrared (VFIR): VFIR supports $16 \mathrm{Mb} / \mathrm{s}$ only. Line coding in VFIR follows a run-length limited
$\mathrm{HHH}(1,13)$ scheme, which guarantees at least one empty chip after each pulse and allows for a maximum of 13 empty chips between pulses. Under the $\mathrm{HHH}$ $(1,13)$ coding scheme, data bit pairs are encoded into three-chip symbols using the state transition table detailed in [11].

- Gigabit Infrared (Giga-IR): Giga-IR supports 512 $\mathrm{Mb} / \mathrm{s}$ and $1.024 \mathrm{~Gb} / \mathrm{s}$. Line coding in Giga-IR is based on the 8010B DC-balanced encoding scheme, in which a data byte is encoded on a 10-bit character, so as to facilitate clock recovery at the receiver and to meet the stringer timing requirements of Giga-IR links. The GigaIR optical transmitter can be either a LED or LD: if a LED is used as the transmitter then the encoded characters are sent over the infrared link using a four-level ASK modulation format, otherwise if a LD is used as the transmitter then the modulation format is a twolevel ASK [12].

IrDA has also provided recommendations on a number of infrared link parameters. In SIR to VFIR speeds the maximum distance between devices should not exceed $1 \mathrm{~m}$ (or $200 \mathrm{~mm}$ for low power transceivers), while the $3 \mathrm{~dB}$ half-width angle ranges between 15 and $30^{\circ}$ at the transmitter and $0-15^{\circ}$ at the receiver. In Giga-IR speeds a docking station is typically used and the maximum distance is limited to $60 \mathrm{~mm}$, while the transceiver angles are also limited to $5^{\circ}$. Moreover, the bit-error-rate that should be achieved for all infrared links is no more than $10^{-8}$ for SIR to VFIR speeds and no more than $10^{-10}$ for Giga-IR, comparing directly to the BERs achieved in modern optical fiber networks. Additional optical and electrical parameters including (a) maximum/minimum optical intensity at the transmitter and optical irradiance at the receiver, (b) pulse rise/fall time, (c) pulse jitter, (d) minimum/maximum pulse duration, and (e) maximum allowable transmission rate deviations may be found in $[11,12]$ for all speeds ranging from SIR to Giga-IR.

The IrDA protocol stack operates above IrPHY and consists of four main transfer protocols: the Infrared Link Access Protocol (IrLAP) [14], the Infrared Link Management Protocol (IrLMP) [15], the Tiny Transport Protocol (TTP) [16], and the Object Exchange (OBEX) protocol [17]. IrLAP is an HDLC-based link layer protocol with a primary function to provide reliable data transfer over half-duplex OW links. Earlier IrLAP implementations (SIR to VFIR speeds) operated in a Go-back$\mathrm{N}$ fashion and maintained frame sequencing information in both directions. Currently, IrLAP is being modified to take full advantage of the bandwidth provided by GigaIR speeds [18], and the next IrLAP standard is expected to provide (a) extended frame size, (b) extended window size, and (c) selective-repeat mode of operation. Apart from reliable data transfer at $\mathrm{Gb} / \mathrm{s}$ speeds, IrLAP has 


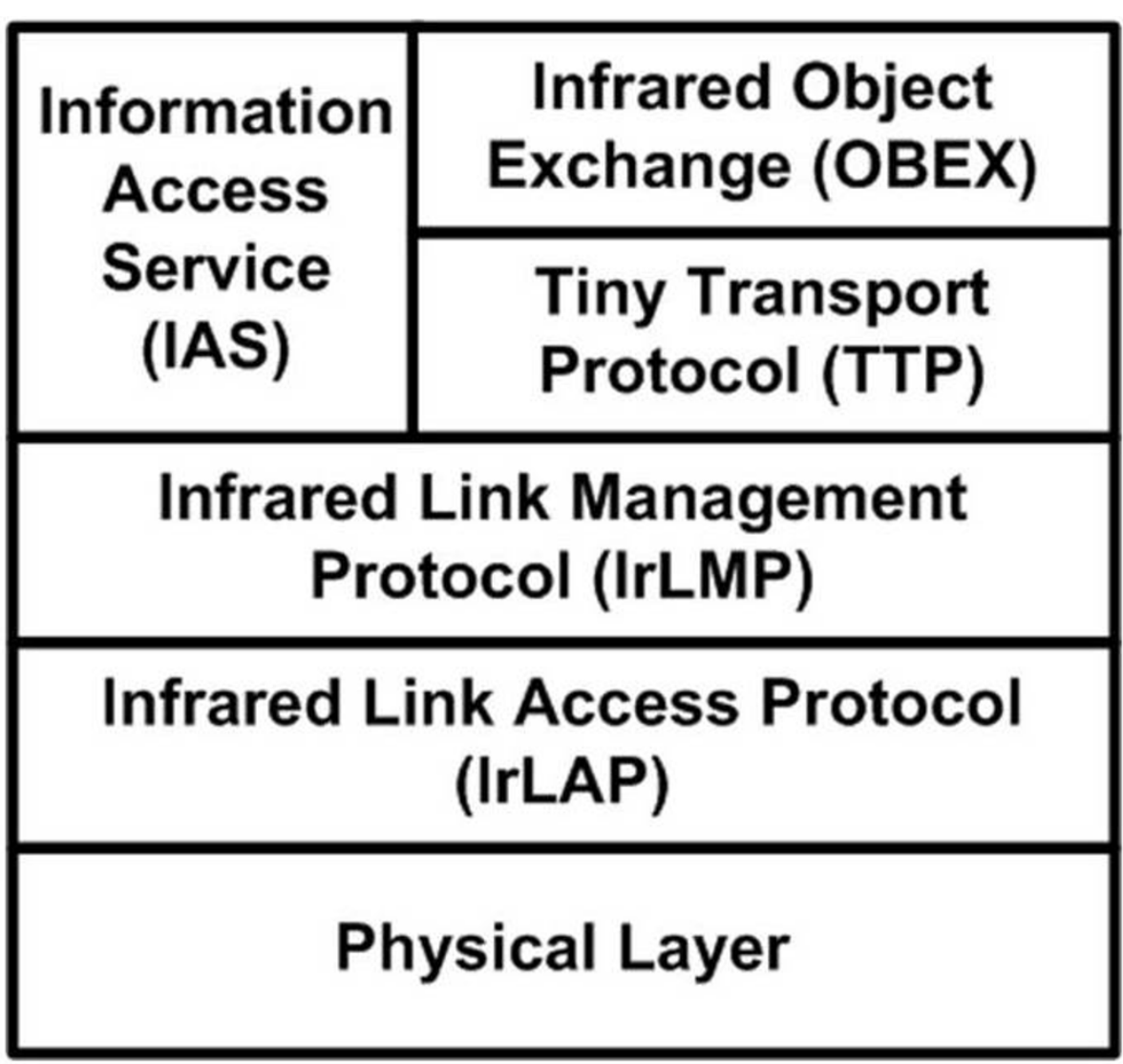

Figure 3 The IrDA protocol stack.

additional features to account for the volatile nature of the infrared physical layer, including extended and dynamic node addressing, link recovery and rapid node discovery (25-300 ms) if fast-connect options have been implemented in IrLAP [19].

IrLMP operates above IrLAP and is responsible for providing upper layers with a multiplexing scheme over IrLAP, as well as a link control mechanism and a service discovery mechanism. IrLMP in fact comprises two separate entities, the Link Management Multiplexer (LM-MUX), which is responsible for link control and data multiplexing and (b) the Link Management Information Access Service (IAS) that maintains information about the services that have been registered at the local station. IrLMP communicates with upper layers and the IAS through multiple Logical Service Access Points (LSAPs). Each LSAP is assigned with a unique identifier that is called the Logical Service Access Point Selector (LSAP-Sel), and source/destination LSAP-Sel pairs are used to multiplex/demultiplex traffic from multiple IrLMP connections in LM-MUX. Moreover, LSAP-Sels of local services are registered in IAS to facilitate service discovery. Remote devices access the IAS by means of a simple command-response protocol (Information Access Protocol-IAP); the LSAP-Sel of IAS itself is well known and equals $0 \times 00$.

TTP is heavily associated with IrLMP and its main functions are (a) to provide per IrLMP connection flow control, and (b) segmentation and re-assembly (SAR) features. Even though IrLAP provides lower level flow control (back-pressure), it is not capable of distinguishing between individual IrLMP flows and as a result using IrLAP back-pressure will slow down all IrLMP connections. TTP on the other hand implements per-connection flow control by associating its own Service Access Points (TTPSAPs) with the LSAPs that are present in the LMMUX on a one-to-one basis. The flow control mechanism itself is credit based: the TTP endpoints issue credits (up to 127 initially) to each other and they use up a credit whenever they send a TTP frame or receive a new credit whenever a TTP frame has been received at the other end. If a TTP endpoint reaches zero credits, it must wait for additional credits to be issued from the other side before sending additional frames.

OBEX is the session protocol that has been accepted by IrDA to ensure interoperability between devices, and 
provide a communication API for user applications. OBEX comprises three main components: (a) a model for representing objects, as well as information about objects, (b) a session protocol that enables session layer communication between devices, and (c) an application framework. Similar to HTTP, OBEX uses a headerbased model to describe both the object attributes and the actual data of the object itself. Session peers, one acting as a client and the other as a server, exchange header information using a request/response communication protocol. Typically, OBEX operates in a stopand-wait fashion, which means that only a single OBEX frame may be outstanding. This behavior limits the infrared link utilization, since requests and responses that involve the transmission of multiple OBEX frames need to wait for an equal number of link turnarounds. In order to mitigate this effect, enhanced OBEX implementations allow for a Single Response Mode (SRM), in which multi-frame requests/responses may be acknowledged by a single frame. Finally, the primary element of the application framework is the default OBEX server. This is the "well-known OBEX server" that is registered in the IAS database and provides basic functionalities like accessing file systems, databases, services and applications. The default OBEX server uses an Inbox service, so that clients can exchange objects using a standard location. Alternatively, clients may also communicate with non-default OBEX servers; in such case multiple OBEX server instances are running along with the applications. The non-default server instances are registered with different LSAP-Sels in the IAS, so that there is no confusion with the default OBEX server.

IrDA has also standardized a stripped-down version of the protocol stack to facilitate implementations in limited capability terminals. This version is commonly known as IrDA Lite and maintains only the most fundamental operations of the protocols involved [20]. A more recent variation of the protocol stack, IrSimple [21], achieves faster transfer speeds by allowing blocks of information to be exchanged over IrLMP. Since the TTP flow control mechanism does not allow for block sizes over 127 frames, TTP is replaced by IrSMP (Sequence Management Protocol for IrSimple) in the IrSimple protocol stack [22]. IrSimple maintains the SAR capabilities of TTP and implements a frame sequencing mechanism to ensure the successful transmission of frame blocks.

IrDA has also provided standards for application related frameworks. One such application framework is Infrared Financial Messaging (IrFM), which supports digital payment applications running on infraredenabled personal communication devices [23]. In IrFM, a personal communication device (also called a Personal Trusted Device-PTD) interacts with a Point of Sale
(POS) terminal. Applications running on PTD and POS communicate over OBEX, with PTD being the OBEX server and POS being the OBEX client. Applications may not access OBEX directly, however, but only through IrFM services. To this end, the IrFM standard defines a number of core services that enable basic transactions between the POS and the PTD, as well as a Core IrFM Protocol (CIP) that passes transaction commands from IrFM services to OBEX. A key feature of IrFM is its fast transaction mechanism (point-and-pay), as a direct result of the point-and-shoot nature of the IrDA protocol stack. In addition, IrFM has been developed to maintain simplicity, security, interoperability and reliability in infrared financial transactions.

Another application framework is "wire replacement" and refers to the replacement of parallel and serial cables with IrDA links. So far there have been two relevant standards, the Serial and Parallel Port Emulation over IR (IrCOMM) [24] and the Infrared Universal Bus (Ir-USB) [25]. IrCOMM defines the emulation of parallel and serial ports over the IrDA protocol stack. The drive between the emulation is to facilitate the migration of applications that have been initially written to work over COM and LPT ports to IrDA compliant devices. To this end, IrCOMM differentiates between four service types: three-wire-raw, three-wire, nine-wire and Centronics. Three-wire-raw involves no control information and the role of IrCOMM is to fill up payload of IrLMP frames with user data. The other three service types, however, require a separate control channel and as a result IrCOMM frames carry control information along with the user data. In addition, IrCOMM interprets flow control commands and utilizes the credit based flow control mechanism of TinyTP in an appropriate fashion. Ir-USB, on the other hand, is mainly based on the USB 2.0 protocol stack and utilizes only IrLAP and IrPHY (Giga-IR in particular) as an ultrafast transmission mechanism. As a result, an operational speed of $480 \mathrm{Mb} / \mathrm{s}$ is provided to USB clients over a 512 $\mathrm{Mb} / \mathrm{s}$ or $1.024 \mathrm{~Gb} / \mathrm{s}$ infrared link. The critical subsystem of the Ir-USB architecture is the Ir-USB controller that acts as an interface between the electronic USB controller and IrLAP. Apart from transmitting data at very high speeds, the Ir-USB controller is also responsible for handling special purpose USB packets, so that the infrared link is essentially transparent to the electronic USB controller.

\subsection{Research-related literature}

OW personal communication systems involve point-topoint links with distances that are typically limited to a few tens of millimeters and very narrow optical beams. As a result multipath dispersion and fading, which severely affect the performance of indoor and outdoor 
systems, respectively, are not present in personal communication systems. The dominant impairment in personal communication systems is optical noise and interference from the sun and artificial lighting [26,27], still the narrow reception angles filter out most of this. The reader may refer to Section III.D for a more detailed discussion on noise and interference, as well applicable mitigation techniques.

The optical channel in personal communication systems is considered to be linear, time invariant and nondispersive $[1,28]$. In practice, there are two main goals regarding the physical layer in personal communications systems: (a) to attain increasingly high link speeds, and (b) to deploy suitable codes that facilitate receiver functions such as clock and frame recovery. To these ends, simple amplitude modulation formats like OOK and amplitude shift keying (ASK) have been previously presented and evaluated [27], even though more complex phase and frequency modulation formats are also possible [29]. Relevant modulation schemes include PPM [30], differential PPM (DPPM) [31], digital pulse interval modulation (DPIM) [32], run-length limited (RLL) coding [33] and DC-balanced coding [34]. PPM is advantageous over OOK for low rate systems, since it requires decreased average power and is more robust against optical noise, especially noise components near baseband resulting from fluorescent lamps [27]. Still, PPM requires a bandwidth increased by a factor of $M / \log _{2} M$ as compared to OOK, where $M$ is the number of chips that are required to construct the PPM symbol. DPIM and DPPM require less bandwidth (approximately half) than PPM and more average power [30], but they also occupy more bandwidth than OOK and ASK. ASK is better suited for higher rate systems; it utilizes the same bandwidth as OOK to provide a $\log _{2} M$ times faster line rate, at the expense of increased average optical power. Table 2 and Figure 4 summarize the bandwidth and BER performance of PPM variants and ASK systems in short-range OW.

RLL codes, on the other hand, are used to alleviate timing uncertainties that cause erroneous data recovery in high-speed PPM receivers. Specifically, the VFIR IrDA $\operatorname{HHH}(1,13)$ run-length limited code performs better clock and data recovery than PPM, while maintaining an acceptable bandwidth efficiency (approximately 67\%) [33]. DC-balanced coding is also receiver oriented and is used to mitigate the baseline wander effect caused by high-pass filtering of optical noise at the receiver [34-36]. More sophisticated modulation schemes that involve transmission over multiple carriers, such as subcarrier modulation (SCM) $[37,38]$ and orthogonal frequency division multiplexing (OFDM) [39-41] are also possible. However, SCM and OFDM are primarily designed to minimize inter-symbol (ISI) interference in indoor systems, and therefore require more complex coding and modulation hardware at the transceivers when compared to OOK.

With respect to protocol analysis in OW personal communication systems, both the initial IEEE 802.11 and IrDA AIr standards have been previously studied in the literature. A performance analysis for AIr may be found in [42-44] where the link-layer frame size is contrasted against the average frame delay and system throughput. IEEE 802.11 has been extensively investigated in the literature, but a complete overview is beyond the scope of this article. Two articles that evaluate the performance of initial 802.11, and apply to OW MAC and PHY are $[45,46]$. As far as the point-andshoot oriented IrDA protocol stack is concerned, numerous research efforts have been presented in the literature. A primary research topic involves the determination of the optimum values that maximize the system throughput for network parameters including (a) the OBEX frame size, (b) the TTP window size, and (c) the IrLAP window size, frame size and link turnaround time. The optimum IrLAP frame and window sizes have been calculated in $[47,48]$ as a function of the link BER, speed and turnaround time. Cross-layer optimization that involves the TTP, however, shows that the IrLAP network parameters are not independent of the TTP flow-control mechanism [49], and this owes to the fact that TTP frame streams are not temporally continuous. Moreover, the Stop-and-Wait fashion in which OBEX operates further complicates the determination of optimum values for the OBEX, TTP and IrLAP network parameters $[50,51]$. Further analysis of the IrDA protocol stack involves several variations: the suitability and scalability of IrSimple for transmission of relatively small files over high-speed OW links is discussed in [52]. An alternative session protocol that is suitable for the transmission of larger files, IrBurst [53], is also optimized against the TTP and IrLAP network parameters in $[54,55]$. Finally, a detailed analysis of IrFM attributes, including its connection delay (transaction speed), simplicity, interoperability, security and reliability, along with a comparison between IrFM and competing technologies may be found in [56].

\section{Indoor OW systems}

OW has been extensively studied as a broadband, low cost and power efficient technology for indoor communication systems [1,27,57-62]. Similar to the case of personal communications, OW indoor systems provide considerable cost savings to device manufacturers and end users due to the existence of unlicensed available spectrum, are perceived as safer than competing RF technologies to humans and do not suffer from RF interference. Indoor OW link architectures, however, are quite 
Table 2 Bandwidth and BER performance of OOK and PPM variants

\begin{tabular}{lll}
\hline Modulation format & Data rate & BER \\
\hline ASK & $R_{b}=B \cdot \log _{2} M$ & $2 \cdot \frac{M-1}{M \cdot \log _{2} M} \cdot Q\left(\frac{1}{M-1} \cdot \sqrt{S N R \cdot \log _{2} M}\right)$ \\
PPM & $R_{b}=B \cdot \frac{\log _{2} M}{M}$ & $\frac{M}{2} \cdot Q\left(\sqrt{\left.S N R \cdot \frac{M \cdot \log _{2} M}{2}\right)}\right.$ \\
DPPM & $\overline{R_{b}}=2 \cdot B \cdot \frac{\log _{2} M}{M+1}$ & Variable symbol duration, refer to [31] for a detailed presentation \\
DPIM & $\overline{R_{b}}=2 \cdot B \cdot \frac{\log _{2} M}{M+3}$ & Variable symbol duration, refer to [32] for a detailed presentation \\
\hline
\end{tabular}

Bandwidth $B$ is the first-null bandwidth, SNR is the electrical signal-to-noise ratio and $M$ is the number of chips that construct a symbol in PPM or the number of power levels in an ASK system. Function $Q(x)$ is defined as $Q(x)=\frac{1}{\sqrt{2 \pi}} \cdot \int_{x}^{\infty} e^{-u^{2} / 2} \cdot d u$.

different from their personal communication counterparts due to the fact that a key requirement here is user mobility. In real-world indoor systems the user is allowed to move within a limited area of coverage, which typically equals the size of a room or an aircraft cabin.

Providing OW access to a moving user can be challenging, since the optical beams are blocked by objects inside the room. A possible solution is to deploy a roof-mounted and narrow-beam transmitter that is controlled by a tracking mechanism. The tracking mechanism rotates the transmitter and directs the narrow optical beam from the transmitter to the mobile receiver. This approach leads to a point-to-point OW link whose architecture is very similar to that of personal communication systems (Figure 5a). An alternative approach is to design the transmitter with a broad-angle emission pattern and allow multiple reflections from the walls and objects inside the room. In such a case, the OW system is categorized as diffuse (Figure 5b). If the emission pattern is properly engineered, the multiple reflections result in uniform illumination inside the area boundaries, even at places that are out of line-of-sight of the transmitter, and therefore enhance user mobility [63]. However, the multipath propagation in diffuse channels leads to time-dispersion of any pulse transmitted on the channel. Multipath dispersion is a critical impairment, since it drastically reduces the system bandwidth, and attainable data rate, and increases the link losses. As a result, point-to-point and diffuse topologies present a tradeoff between mobility and bandwidth. More advanced topologies, for instance multispot diffusing (Figure 5c), combine the best of both worlds and allow for significant user mobility while at the same time achieve two orders of magnitude higher bandwidth than purely diffuse ones. All three topologies (point-to-point, diffuse, and multispot diffusing) are discussed in further detail in Sections 3.1-3.3, and a summary of their respective strengths and weaknesses is given in Table 3.

Another major difference between personal communication and indoor systems is the impact of noise on their performance. Indoor OW receivers are typically designed with broader reception angles and as a result the received optical noise power levels are higher when compared to those of personal communications. Noise originating from the sun and artificial lighting interferes with the optical signal and degrades the signal quality at a baseband frequency range between DC and tens of $\mathrm{MHz}$. This is unfortunately the operational frequency range for most indoor OW systems. Filtering techniques combined with modulation formats are required to reject a significant percentage of the received noise power and to limit the noise induced performance degradation. Noise sources, their impact on indoor OW and mitigation techniques are discussed in Section 3.4.

Finally, apart from discussing system requirements and key impairments in indoor OW systems, this section also presents the latest trend in indoor OW: VLCs. The main idea behind VLCs is to use solid state LEDs, which were originally developed for indoor lighting, for communication purposes, as well. VLCs are particularly appealing since they do not require additional transmitters and receivers, as is the case in infrared communications, and since ceiling LED installations are already designed to provide a uniform illumination pattern of high optical power within the area of coverage [64]. VLCs are presented in Section 3.5.

\subsection{Point-to-point links}

Point-to-point links require a line-of-sight (LOS) between the transmitter and the receiver, as shown in Figure 5a. Such a link topology permits the use of highly 

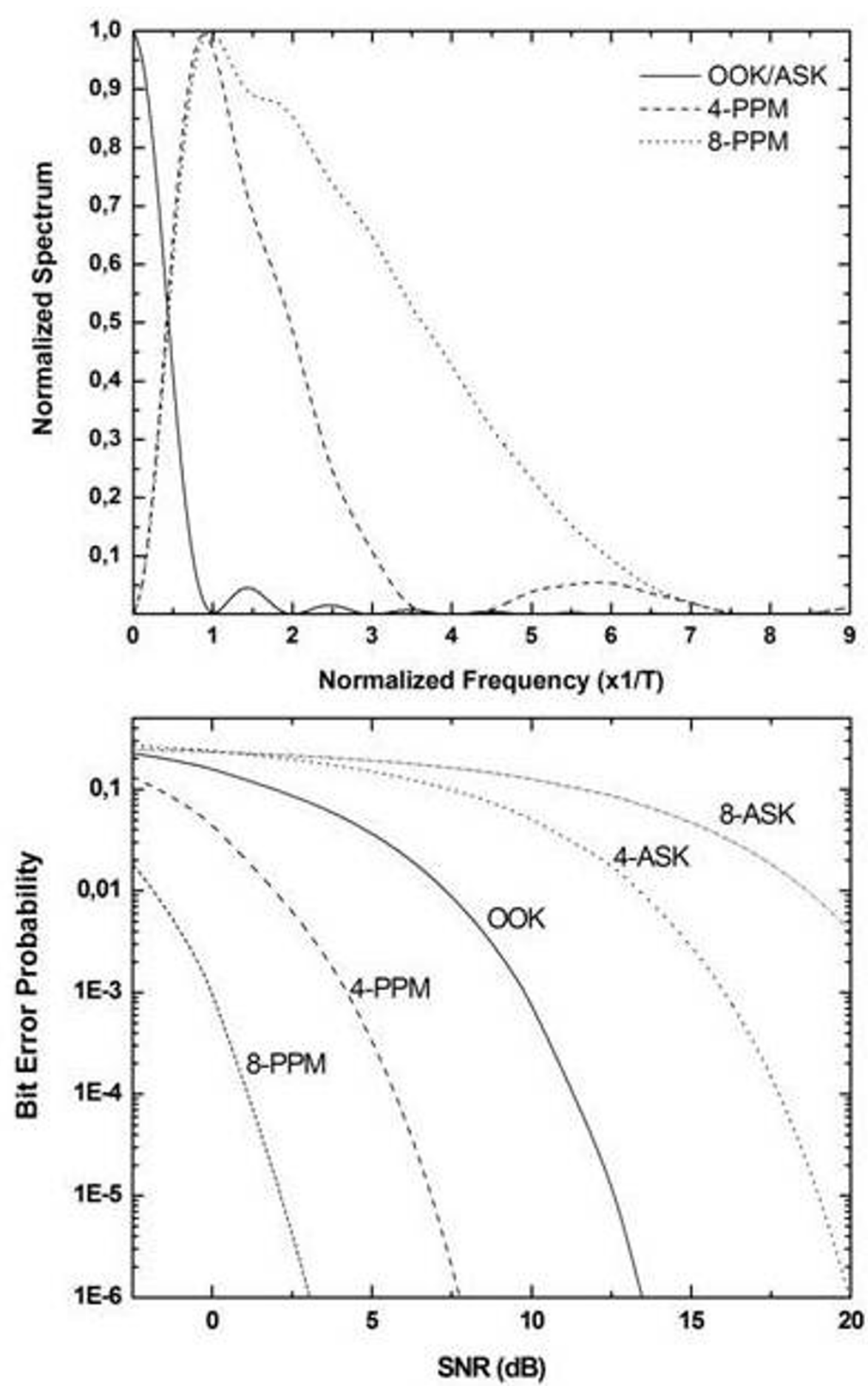

Figure 4 Spectral properties and BER performance of PPM and OOK/ASK systems, following the analysis of [60].

directional transmitters with low beam divergence and narrow field-of-view (FOV) receivers. Thus, point-topoint links are able to reject the majority of ambient light that interferes with communications. In addition, the directionality of the link removes the impact of multipath dispersion since any reflected transmitter energy is essentially rejected by the receiver. Still, a disadvantage of this link topology is the fact that precise alignment and pointing are required, making the realization of mobile links difficult. 


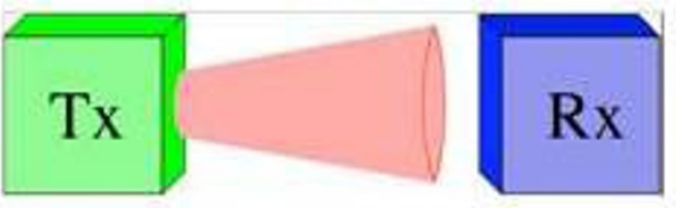

(a) Point-to-point

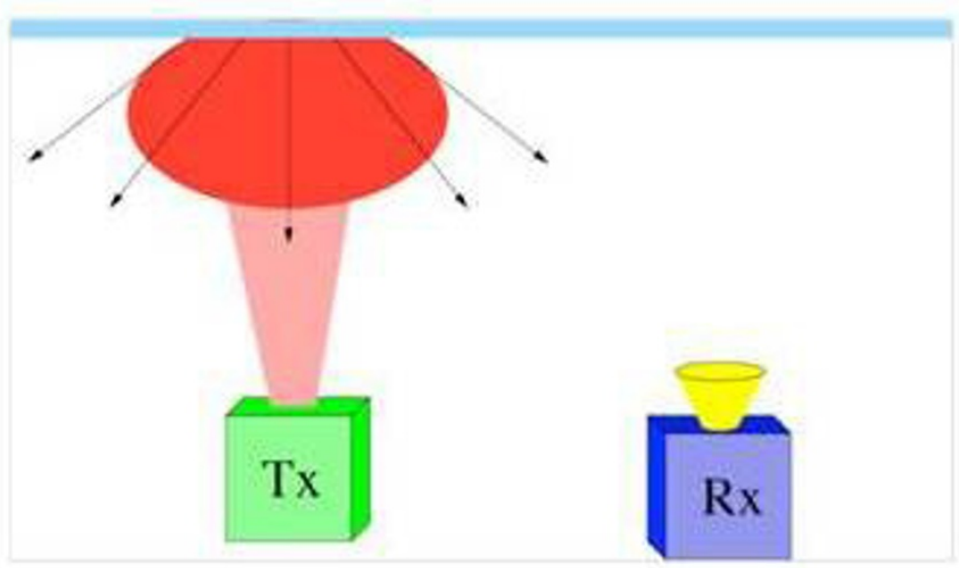

(b) Diffuse

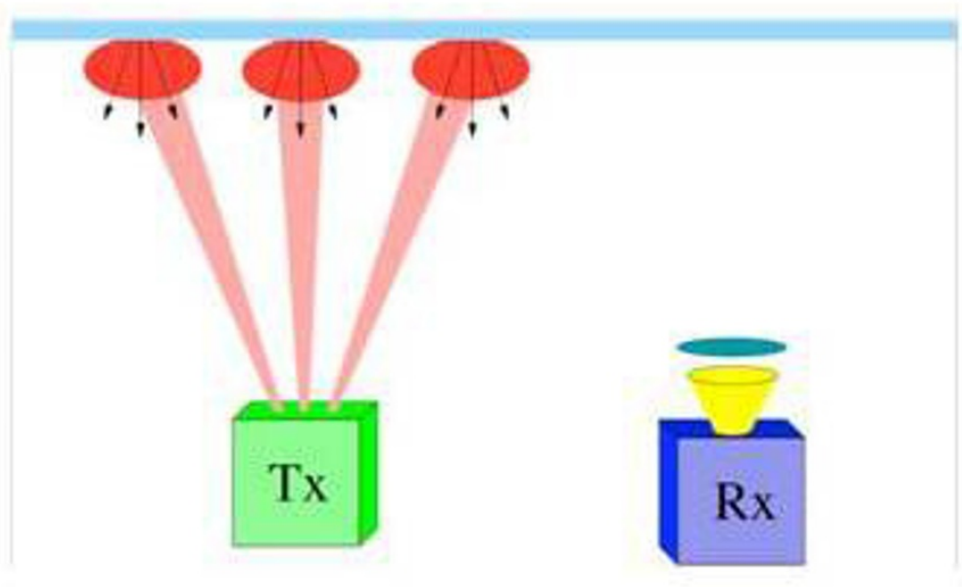

(c) Multispot Diffusing

Figure 5 Topologies for indoor optical wireless systems. (a) Point-to-point, (b) diffuse, (c) multispot diffusing.

Very high data rates can be achieved in point-to-point indoor OW links by means of spatial division multiplexing, a technique that has been considered for some time. In [57], hundreds of parallel links, each operating at $50 \mathrm{Mb} / \mathrm{s}$, were shown feasible in large areas using a ceiling-mounted transmitter. The transmitter was capable of creating a large number of narrow beams pointed to a variety of locations. Although such systems are ideal for high-speed data distribution, they are of high complexity and do not permit motion of terminals. 
Table 3 Comparison of OW link topologies for indoor systems

\begin{tabular}{|c|c|c|c|}
\hline & \multicolumn{3}{|l|}{ Link type } \\
\hline & $\begin{array}{l}\text { Point-to- } \\
\text { point }\end{array}$ & Diffuse & $\begin{array}{l}\text { Multispot } \\
\text { diffusing }\end{array}$ \\
\hline Link rate & High & Moderate & Moderate \\
\hline $\begin{array}{l}\text { Implementation } \\
\text { complexity }\end{array}$ & Low & Low & High \\
\hline Beam pointing & $\begin{array}{l}\text { Required } \\
\text { (exact) }\end{array}$ & $\begin{array}{l}\text { Not- } \\
\text { required }\end{array}$ & Required (partial) \\
\hline Beam blocking & Yes & No & No \\
\hline User mobility & Limited & High & High \\
\hline Dispersion & None & High & Low \\
\hline Path loss & Low & High & Moderate \\
\hline $\begin{array}{l}\text { Impact of ambient } \\
\text { noise }\end{array}$ & Low & High & Low \\
\hline
\end{tabular}

High-speed electronic tracking was reported in [65] to support mobility at a rate of $155 \mathrm{Mb} / \mathrm{s}$ over ranges of nearly $2 \mathrm{~m}$. Tracking was accomplished by using a transmitter that had an array of laser diodes combined with optics and a receiver with a wide FOV and detection array. Tracking involved the selection and switching of paths onto the appropriate receive element.

Point-to-point OW links have also found a great deal of popularity in a variety of shorter-range applications. As an example, the IrDA IrFM specification permits secure and quick payment options at point-of-sale terminals [56], as has been detailed in Section 2. Another example is a system provided by Talking Signs, which is able to deliver voice to visually impaired individuals using fixed infrared transmitters and hand-held receivers. Yet another area of application that has been active for some time is the use of OW links for high-speed backplanes. High bandwidth communication between circuit boards is a major limitation in many large computing and communication platforms. Using an array of tightly aligned transmitters and receivers, high-data rate links that are free from the capacitive, EMI and bandwidth limitations of on-board links [66,67] can be established. An OW backplane has also been proposed to provide intra-craft data connectivity in a spacecraft [68].

The majority of indoor point-to-point OW links have single element transmitters and receivers. However, great gains can be attained by using multiple-transmitters and receivers in a coordinated fashion. The implementation of such Multiple-Input-Multiple-Output (MIMO) links involves the use of laser and photodiode arrays [69], optical interconnects [70] or a spatial-light modulator (SLM) as a transmitter and an imager as a receiver. Incoming data is either parallelized and transmitted over multiple beams, while when a SLM is employed, data is sent by creating a series of twodimensional (2D) optical intensity images. OW MIMO links require tight spatial alignment between the transmitter and receiver arrays to avoid interference between the channels [71-73]. Recently a spatial processing technique that does not require tight spatial alignment has been developed for OW MIMO links [74,75]. In this technique, the interference between spatial channels is modeled and used in communication system design directly to provide high spectral efficiency. Alternatively, interference can be minimized by allowing only a single laser element to transmit, as proposed in [76], or by using interference cancelation techniques at the receiver [77].

MIMO links have been used in a variety of applications including 2D barcodes $[78,79]$, page-oriented recording $[80,81]$, holographic storage $[71,72,82,83]$, and MIMO OW communications [27,66,84,85]. With respect to the 2D barcodes, OW MIMO links are currently used on a wide array of cellular telephones in the form of barcode readers. Data are encoded as a 2D barcode which is either printed or displayed on a billboard. A cell phone camera is then used to image the barcode and processing is done to extract the data. Many newspapers now publish 2D barcodes which, when imaged by a cell phone camera, direct the cell phone browser to the appropriate webpage for additional details on the story [86].

\subsection{Diffuse indoor OW links}

Initial studies into diffuse OW links were presented in 1979 [1] and diffuse links were shown to be robust, immune to shadowing and allowed for terminal mobility. Unlike point-to-point OW links, a diffuse OW link does not require the existence of a LOS to establish communications. Rather, as illustrated in Figure 5b, it employs wide beam transmitters and large FOV receivers to exploit diffuse reflections from the walls and objects inside the room and establish a communication link $[1,27,87,88]$. As a result, there is inherent path diversity and diffuse links are relatively immune to blockage and pointing errors and permit a great degree of mobility for the receivers. However, the received signal is corrupted by multipath dispersion due to the large number of collected reflections at the receiver. The temporal spread of reflections results in inter-symbol interference at high data rates and limits the system bandwidth. Notice that although diffuse OW links suffer from multipath dispersion, they do not exhibit multipath fading as in RF links. The detector in diffuse OW links typically has a size of many thousands of wavelengths and the received optical intensity is integrated over the surface of the detector and provides an inherent degree of spatial diversity [27]. Thus, the channel characteristics are unaltered when moving the detector by many wavelengths. 
The indoor diffuse OW channel is well modeled as a linear, time-dispersive channel with a time-invariant impulse response $h(t)$ [89]. The impulse response is calculated from the individual reflections of walls and objects inside the room and there is an array of methods in the literature to compute the impulse response in optical intensity domain for a typical room layout. These methods include ray tracing $[89,90]$, experimental measurements $[28,91,92]$, simplified functional modeling [93,94], photon tracing [95] and Monte Carlo ray tracing methods [96-99]. While specular reflections are possible, the vast majority of reflections are diffuse since the dimensions of the surface roughness is on the order of the wavelength of light used in the link [95]. As a result, the optical power is reflected nearly independently of the angle of incidence of the optical wave. The intensity distribution of an individual diffuse reflection is well approximated by a Lambertian pattern $[1,60,89]$.

The impact of multipath dispersion on diffuse indoor OW links is quantified by the path loss and normalized delay spread. The path loss is calculated from the channel impulse response $h(t)$ and ranges from $10^{-7}$ to $10^{-5}$ $[27,28]$. The temporal dispersion due to multipath can be measured by the root-mean square (RMS) delay spread of the impulse response and ranges from 2 to 10 ns $[27,28]$. A popular and convenient model that correlates the temporal dispersion with the channel bandwidth is the exponential-decay model $[93,94]$. In this model, the $3-\mathrm{dB}$ bandwidth of the channel impulse response is calculated from the channel temporal dispersion $D$ using the following simple analytical relation

$$
f_{3 \mathrm{~dB}}=\frac{1}{4 \pi D}
$$

A range in temporal dispersion $D$ from 2 to $10 \mathrm{~ns}$ $[27,28]$ implies that the $3-\mathrm{dB}$ channel bandwidth approximately ranges from 10 to $40 \mathrm{MHz}$. The impulse response, delay spread and path loss depend on the particular composition and arrangement of the room and are considered fixed once the room characteristics are fixed [27].

Multipath dispersion severely limits the diffuse channel bandwidth and a vast array of modulation, coding and equalization techniques have been applied to combat this effect $[27,89,100-103]$. An important aspect of these techniques is that amplitude constraints must also be imposed on all signals transmitted on OW channels, since the optical power signal must satisfy a non-negativity constraint. In addition, due to eye- and skin-safety requirements a limitation on the average optical power is also mandatory as imposed by international eye-safety limitations [13]. This power limitation is in stark contrast to the mean squared constraint in RF channels. As a result, the use of modulation and coding for RF channels directly on OW channels is seldom efficient and the unique power and bandwidth efficiency requirement of the OW channel must be considered in any communication system design [60].

Diffuse OW links have been standardized by both IrDA and IEEE. The standardization of diffuse OW links from IrDA resulted in the Advanced Infrared (AIr) physical layer interface that defines the operation of $4 \mathrm{Mb} / \mathrm{s}$ diffuse OW links inside typical room sizes [2,43,44,104]. IEEE included a physical layer specification for infrared OW communications at 1 and $2 \mathrm{Mb} / \mathrm{s}$ in its 802.11 standard. Experimentally, diffuse infrared OW links have been demonstrated at $25 \mathrm{Mb} / \mathrm{s}$ [105] and $50 \mathrm{Mb} / \mathrm{s}$ $[106,107]$.

\subsection{Multispot diffusing links}

Spatial diversity techniques have been successfully applied to indoor OW links in a topology that is termed multispot diffusing (MSD) and shown in Figure 5c[108]. The MSD architecture aims to combine the advantages of point-to-point links with the mobility of diffuse links [108-115]. To this end, the MSD transmitter modulates data onto a series of beams that are projected onto the ceiling above the communications floor. The MSD receiver ideally images one or perhaps several spots and decodes data from the diffusely reflected energy. The same data is modulated for all spots and the arrangement and number of spots is optimized so that at least one spot is in the imager for every receiver position.

Even though both diffuse OW and MSD links rely on diffuse reflections on surfaces inside the room, there are large differences in the underlying channel models of both topologies. The first main difference lies in the intensity distribution of the optical transmitter. Whereas diffuse OW links emit light over a large divergence angle, the MSD transmitter uses a series of narrow divergence beams directed to the ceiling. This more efficient organization of the optical intensity leads to a far smaller path loss in MSD systems than in diffuse systems. The second major difference lies in the construction of the receiver. The MSD receiver consists of a series of narrow FOV elements directed to the ceiling. These narrow FOV receivers reject a large portion of ambient light. In addition, the narrow FOV receivers are able to reject any stray multipath signals and this effect significantly improves the bandwidth of the channel. It has been shown that in a typical room setting the channel bandwidth of an MSD system is in excess of $2 \mathrm{GHz}$, which is in stark contrast to the tens of $\mathrm{MHz}$ available in diffuse systems [116].

One of the challenges in implementing an MSD link is the creation of an array of spots on the ceiling. 
Although straightforward, using arrays of emitters with their own optics is bulky and expensive [115]. A less expensive and compact method is to use a laser diode and a computer generated hologram. In [112], a hologram which produces an array of $8 \times 8$ beams is presented, while holographic diffusers with fewer lobes were considered earlier in [63]. Holograms have also been considered to create arrays as large as $10 \times 10$ spots [117]. Other work has considered different geometries for MSD spots including a line strip and a diamond in an effort to reduce transmitter complexity $[118,119]$. Experimental work on MSD systems has also shown promising results. An angle diversity receiver composed of discrete elements working at $70 \mathrm{Mb} / \mathrm{s}$ at a BER less than $10^{-9}$ over a 4-m range was reported in [115]. In [114], system issues for a $100 \mathrm{Mb} / \mathrm{s}$ MSD link were also presented. A $140 \mathrm{Mb} / \mathrm{s}$ MSD system with a single receiver [120] and a $155 \mathrm{Mb} / \mathrm{s}$ using a multi-element imaging receiver are presented in [65]. Following the above, MSD links maintain the high bandwidth and low path losses of point-to-point links and at the same time achieve mobility that is comparable with those in diffuse links at the expense of slightly more complicated transmitters and receivers [112]. As a result, the MSD architecture is an excellent topology for future indoor highspeed content distribution systems.

\subsection{Noise and interference modeling and mitigation}

Noise and interference are two additional impairments that affect the performance of indoor OW links. In typical indoor diffuse OW systems, which involve intensity modulation and direct detection (IM/DD), a good model that describes noise and interference is the baseband model

$$
y(t)=R \cdot x(t) * h(t)+z(t) .
$$

$x(t)$ is the transmitted optical power, $y(t)$ is the current at the receiver, $h(t)$ is the optical channel impulse response, $R$ is the receiver photodiode responsivity and * denotes continuous time convolution. The signal $z(t)$ represents noise and interference added at the receiver. In general, $z(t)$ is a high intensity shot noise process. Such processes are well modeled as zero-mean, Gaussian distributed, independent of $x(t)$ and white [27]. However, this model is only true under the assumption of intense ambient light. For example, in links with low background illumination a photon-counting model is more appropriate [60].

The sources of noise and interference include background illumination, lighting from incandescent and fluorescent sources as well are front-end circuit noise in the preamp of the receiver $[1,26,27,60,87,121,122]$. Figure 6 summarizes the spectral distribution of solar, incandescent, and fluorescent radiation in the visible and near- infrared wavelength ranges, which are typically employed in OW systems. The sun emits broadband un-modulated light that is converted to shot-noise current at the receiver. The root-mean-square (RMS) of the noise current is proportional to the incident optical power and the receiver bandwidth and band-limited optical and electrical filters at the receiver can reject a significant portion of the shot noise. Incandescent lamps are modulated by their AC power supply $(50 / 60 \mathrm{~Hz})$ and produce a narrowband photocurrent with a total bandwidth of less than $2 \mathrm{kHz}$ [122]. In practical OW systems the receiver bandwidth is significantly higher than $2 \mathrm{kHz}$ and the photocurrent from incandescent light is modeled as a constant RMS shot noise current that is added to the photocurrent from sunlight [27]. Fluorescent lamps, on the other hand, emit light that is modulated at the power supply frequency $(50 / 60 \mathrm{~Hz})$ or higher frequencies (tens or hundreds of $\mathrm{kHz}$ ). Light from $50 / 60 \mathrm{~Hz}$ fluorescent lamps produces a photocurrent with a total bandwidth of 20 $\mathrm{kHz}$ [122], while higher modulation frequencies result in photocurrents that have a considerable part of their power spectrum in the $\mathrm{MHz}$ range [121]. Consequently, the bandwidth of the photocurrent from fluorescent lamps is a significant percentage of the total bandwidth in practical OW systems and the photocurrent is modeled as a time-varying interference signal [27].

The effects of fluorescent light are particularly deleterious for OOK/ASK modulation formats, in which the electrical power spectrum of OOK/ASK contains a strong DC component. Fluorescent noise cannot be filtered without rejecting a significant portion of the signal in low rate OOK/ASK systems [121], and adaptive filtering [123-125] or spread spectrum [126] techniques are required. Higher rate OOK/ASK systems have a broader spectrum and fluorescent noise may be rejected through high pass filtering, however this leads to a baseline wander effect and additional DC-balanced line coding is required [35]. Low rate PPM systems and their variants (DPIM, DPPM) are less vulnerable to noise from slowly modulated fluorescent lamps than OOK/ASK systems, since the peak spectrums of PPM is located at non-zero frequencies and high-pass filtering rejects fluorescent noise without imposing significant ISI [121]. Rejecting fluorescent light modulated at higher frequencies, however, requires processing techniques similar to those used for OOK/ASK [123]. Finally, SCM and OFDM systems may avoid fluorescent noise altogether by utilizing transmission frequencies that are located outside the fluorescent noise bandwidth.

\subsection{VLC systems}

With the advent of solid-state lighting based on white LEDs, there is intense interest in the use of visible light 


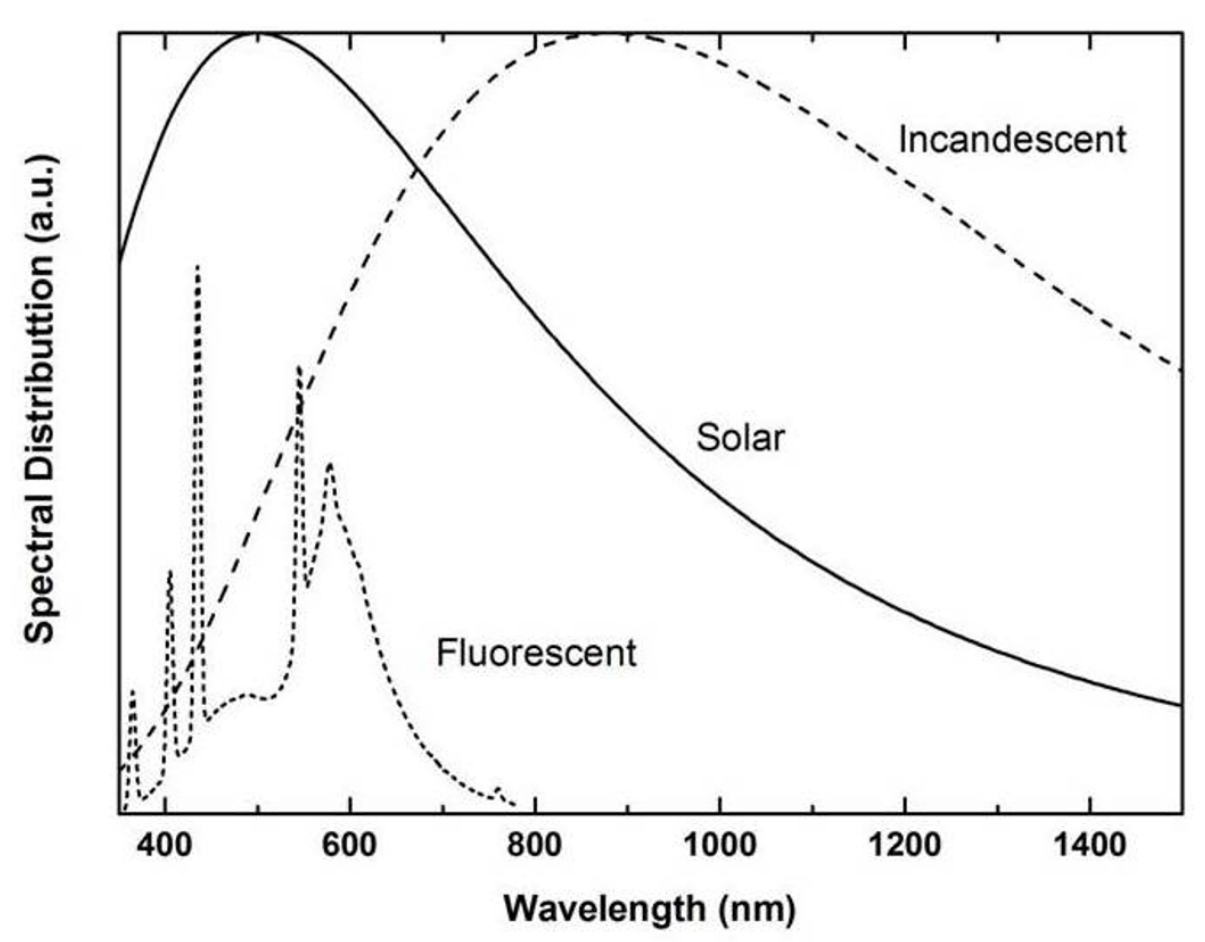

Figure 6 Spectral distribution of solar radiation and emissions from incandescent/fluorescent lamps in the visible and near-infrared wavelength range. Solar and incandescent spectra are calculated from Wien's displacement law at temperatures of 5800 and $3300^{\circ} \mathrm{K}$, respectively. Fluorescent spectrum corresponds to a commercially available lamp.

for OW communications. Not only do white LEDs for illumination purposes have high energy efficiency and lifetimes, but they also possess significant modulation bandwidth. By modulating the illumination outside of the human perceptual range, significant data rates can be transmitted while satisfying the illumination requirements inside a room $[59,127]$. Such VLC channels also possess an SNR that is approximately $30 \mathrm{~dB}$ higher than a comparable infrared link since the background interference is largely removed [128]. Moreover, it is not necessary to install additional LEDs for telecommunication purposes. Visible light LEDs are already designed to provide uniform indoor lighting, which means that the effects of shadowing are minimized. In their simplest implementation VLCs only provide broadcast download capability, since the LED lighting will not in general include optical receivers.

VLCs have drawn significant attention from standardization bodies and research institutes in Europe, the USA and Japan. In Japan, VLC standardization efforts have been underway for some time. The VLC consortium [3] was founded in 2003 to investigate and provide a standard platform for VLC systems. In 2007, the VLC standard JEITA CP-1221 was issued by the Japan Electronics and Information Technology Industries Association [129]. European efforts in VLC have also been significant. The OMEGA project [130], funded by the European Commission [131], is tasked to develop the physical channels to enable $\mathrm{Gb} / \mathrm{s}$ rates for home area networks combining RF, VLC, infrared and power line communications. A total of 20 partners are included in this project which has a budget on the order of $€ 19$ million and a European Commission fund of $€ 12.4$ million [132]. In the USA, an NSF research fund of $\$ 18.5$ million has been provided to three universities to establish Smart Lighting Engineering Research Centers, which will develop device and system technologies for VLC communications. In addition, standardization efforts for VLC through the IEEE have been quite active recently [133].

Similar to infrared OW, the transmission rates of VLC systems are limited by the effects of multipath dispersion and noise from the sun and artificial lighting. In principle, the same techniques that have been presented in previous paragraphs to mitigate dispersion and noise may be applied to VLCs. As a result, multiple carrier modulation formats (SCM [134] and OFDM $[135,136]$ ), MIMO systems [137], coding schemes [138,139], and adaptive equalization methods [140] have been proposed in the literature within the context of VLCs. The transmission rates of VLC systems are also limited by the modulation bandwidth of phosphor-based visible LEDs; 
the slow time constant of the phosphor they are manufactured with in order to emit yellow light leads to a typical modulation bandwidth of a few MHz. Filtering and equalization at either the transmitter [141] or the receiver [142] alleviates the slow phosphor response, and increases the visible LED bandwidth by an order of magnitude. This bandwidth increase, along with the use of discrete multi-tone OFDM, has enabled the demonstration of VLCs operating at transmission rates that exceed $100 \mathrm{Mb} / \mathrm{s}$ [143-145].

An additional aspect of VLCs is that visible LEDS are also required to provide lighting at a constant power level and brightness adjustment options. The human eye is not capable of distinguishing between power variations at the bit level for typical transmission speeds under consideration in VLC systems (tens to hundreds of $\mathrm{Mb} / \mathrm{s}$ ). However, long series of '1's and ' 0 's lead to long-term power variations that are perceived as flickering. Flickering is harmful for the human eye and several approaches, which aim at maintaining a constant DCcomponent in the emitted VLC signals, have been taken to mitigate its effect. The most common mitigation technique is to use PPM, in which transmitted symbols maintain a pre-determined percentage of ' 1 's and '0's. A performance analysis of PPM variants may be found in $[134,146]$. Another approach involves using DCbalanced line coding such as modified $4 \mathrm{~b} 5 \mathrm{~b}, 5 \mathrm{~b} 6 \mathrm{~b}$ or 8b10b over OOK [147]. As far as brightness adjustment is concerned, it is accomplished by combining line coding with pulse-width-modulation (PWM) variants $[147,148]$. Under this scheme, line coding provides a DC-component whose amplitude can be adjusted by varying the duty cycle of the PWM.

\section{Outdoor OW systems}

Another major application area of OW is in outdoor systems. From a commercial perspective, outdoor OW complements optical fibers in LAN interconnections and broadband access networks, providing a high-bandwidth and low-cost alternative [149]. The installation of an outdoor OW system alleviates the most significant expenditure of fiber deployment: the digging up and repairing of roads. In addition, current commercially available systems operate at $10 \mathrm{~Gb} / \mathrm{s}$ Ethernet speeds, which are comparable to the speeds attained per channel in commercial optical fiber networks.

Directional OW links also have a low probability of intercept and detection compared to broadcast or lessdirectional RF wireless links. These are desirable attributes for certain applications, especially military communications, where the communicating parties need to remain undetectable by an adversary. In contrast to RF transmissions, where the adversary needs only a low complexity receiver to detect broadcast transmissions or the side-lobes of directional RF antennas, implementation of an OW link in a completely covert fashion requires fully optical pointing, acquisition and tracking without any adjunct RF technology from the third party. Thus, it is essentially impossible for the third party to detect the existence of the link, unless they insert their own receiver into the beam path. The security provided by the optical link is enhanced by means of encryption, which ultimately prevents interception of the actual data being transmitted.

Two key requirements for outdoor OW systems are ever-increasing line speeds and link distances. Multi$\mathrm{Gb} / \mathrm{s} \mathrm{OW}$ line speeds are feasible at modest distances at the time of writing, owing to technological advances in optical fiber technology and adaptive optics. Fiber networks are expected to advance to $100 \mathrm{~Gb} / \mathrm{s}$ in the near future, providing the necessary end-point components for OW, as well. As a result, OW line speeds are expected to keep up with their fiber optic counterparts, attaining ultra-high capacities per channel.

In ultra-long range outdoor OW systems, however, the achievable data rates can be severely limited by the earth's atmosphere. The atmosphere has a detrimental impact on optical beams, an impact that worsens as the link length increases [150]. The effects that come into play in making the outdoor OW propagation channel non-ideal are shown schematically in Figure 7. Obscuration (haze, fog, snow, rain) along the path reduces the received power. Even in the absence of significant obscuration, atmospheric turbulence presents significant challenges in the operation of OW links for distances as short as $500 \mathrm{~m}$. Turbulence causes fluctuating intensity at the received power and beam wandering, spreading or breakup. The net result of these effects is manifest as scintillation in the received optical power. Scintillation presents a challenge for the receiver in an OW link, since the receiver must have significant dynamic range, which may need to be more than $40 \mathrm{~dB}$, to handle large variations in the received intensity. Reductions in the received power that take the receiver below the specified threshold correspond to fades. During a deep fade, receiver and background noise dominate over the received signal, rendering the link useless.

The stochastic nature of the fades and surges, both in terms of their intensity and their duration, leads to a stochastic description of the channel response. This is in stark contrast with personal communication and indoor systems, where the channel impulse response is modeled as time invariant. Stochastic channel models suitable for outdoor OW are detailed in Section 4.1. Moreover, due to the stochastic nature of the outdoor OW channel, mitigation of channel-imposed degradations is challenging. Applicable mitigation techniques involve some form of diversity, either spatial or temporal, to increase 


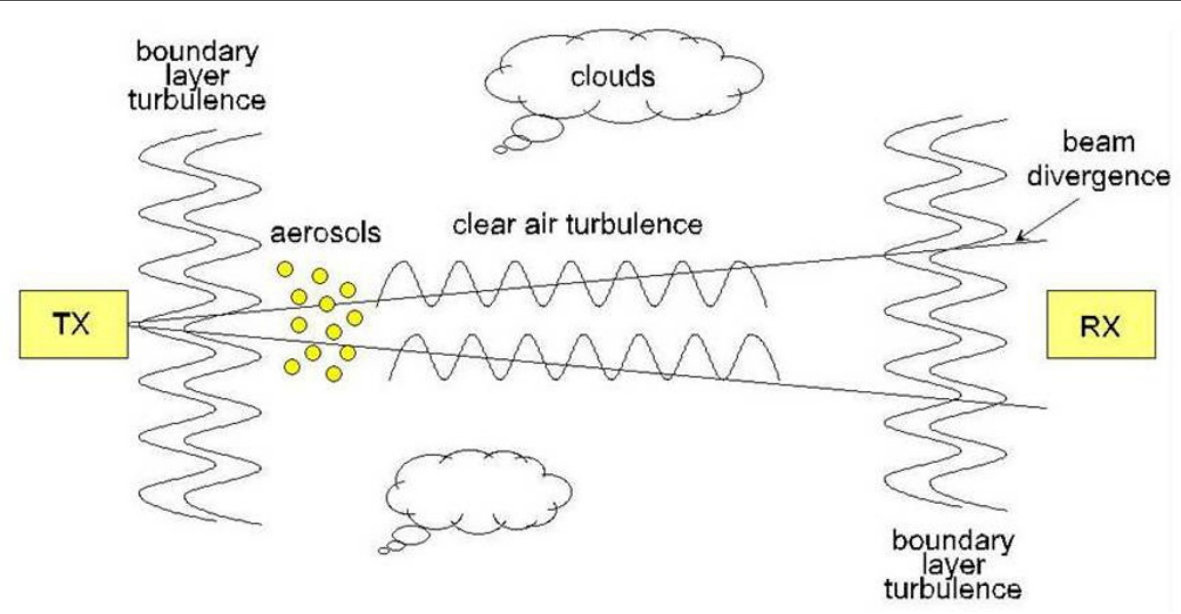

Figure 7 Factors affecting the communication channel for outdoor FSO links.

the probability of signal reception. This is accomplished by sending multiple replicas of the original signal over different propagation paths (spatial diversity) or by temporally displacing replicas by an offset that exceeds the fade duration (temporal diversity). Error-correction-coding is also applicable, but in high capacity systems the number of bits affected by a fade increases with the line rate. Still, these techniques can easily add several seconds of latency to a link. In the $\mathrm{Gb} / \mathrm{s}$ regime, the code length that is required to correct the erroneous bits might be impractically long. Mitigation techniques are discussed in Section 4.2.

The section concludes with the most challenging area of application of outdoor OW systems, satellite communications. These systems employ laser beams that communicate data between terrestrial feeders, satellites and airborne relays and involve link distances that reach up to thousands of kilometers. The impact of atmospheric effects is magnified for such distances, while highly accurate beam acquisition, pointing and tracking systems are required to maintain a working link. OW for satellite communications is presented in Section 4.3.

\subsection{Channel modeling}

A major effect of the atmosphere on optical beams is the rapid attenuation of their power when they propagate through obscurations (haze, fog, snow and rain). For a single mode Gaussian beam of small angle beam divergence $\theta$ and power $P_{T X}$ at the transmitter, the received power at an aperture of area $A$ at range $L$ equals

$$
P_{R X}=\frac{2 P_{T X} A e^{-\alpha L}}{\pi \theta^{2} L^{2}}
$$

where $\alpha$ is the intensity attenuation coefficient along the obscured path. Typical obscuration losses that are encountered near the ground range from $0.5 \mathrm{~dB} / \mathrm{km}$ in clear air, $3 \mathrm{~dB} / \mathrm{km}$ in haze, $50 \mathrm{~dB} / \mathrm{km}$ in thick fog, and $350 \mathrm{~dB} / \mathrm{km}$ in impenetrable fog (OW links are more affected by snow and fog than they are by rain). At high altitude in clear air the attenuation may be as low as 0.1 $\mathrm{dB} / \mathrm{km}$, although aerosols can still affect the channel [151]. In practice, the exponential decay of the optical power means that OW links do not work over significant distances through obscuration and it is not practical to attempt to operate OW links through significant obscuration by increasing transmitter power. Some additional range can be accomplished by decreasing beam divergence angle $\theta$, although this imposes more rigorous pointing and tracking requirements on the link.

A second performance degradation in outdoor OW systems results from the rapid scintillation of the received power caused by atmospheric turbulence. The local density of the atmosphere is constantly fluctuating because of temperature and pressure fluctuations. When a laser beam propagates through the atmosphere, the randomly varying spatial distribution of refractive index that it encounters causes the following effects [152]:

(a) Fluctuating intensity as observed with an optical detector at the end of the path (scintillation).

(b) A varying degree of intensity fluctuation that depends on the receiver aperture (the size of the detector, or the size of the receiving optics which direct the collected light to the detector).

(c) Progressive deterioration of circularly symmetric Gaussian beams with increasing distance and turbulence strength. The progressive changes that are observed are time-dependent deviations of the beam shape from circular, wander of the centroid of the beam, increase in the width of the beam over and above that expected from diffraction, and breakup of the beam into distinct 
patches of illumination whose shapes and locations fluctuate with time.

(d) A fall in the coherence length of the laser beam.

(e) Fluctuations in the angle of arrival of the phase fronts at the receiver.

Theoretical analysis of the effects of the turbulent atmosphere on the propagation of electromagnetic waves has been extensive, and to some extent repetitive, over at least the last 40 years. A substantial amount of this study was carried out in the former Soviet Union, and the books by Tatarski [153] and Chernov [154] are classic accounts of the state of the field at the beginning of the 1960s. Comprehensive reviews of the specific situation regarding laser beam propagation have been given by Ishimaru [155], Strohbehn [156], Zuev [157], Andrews and Phillips [152], and Andrews et al. [158]. There are also a number of other valuable review articles, for example $[159,160]$. Many hundreds of additional articles covering the effect of turbulence on propagating laser beams can be found in [152].

The turbulence induced scintillation at the receiver is characterized by the probability density function (pdf) of the received power. The pdf allows the calculation of the probability of fades or surges of a given level below or above the average received power. The pdf can also be used to estimate the fraction of time that a given link will be in a fade or surge state. It is frequently found experimentally that over a wide range of scintillation indices the distribution of $\log$ field amplitude is a normal distribution, as are the log intensity variations measured at a point [156]. For log-normal statistics the probability of a fade (or surge) of a given depth can be calculated using the methods described by Yura and McKinley [161], who also provide a means to calculate the mean duration of a fade. An alternative pdf that better describes the distribution of received power in strong turbulence is the $\gamma-\gamma$ distribution $[157,159,162,163]$. If scintillation is reduced by aperture averaging, which is likely for all but very long links, then log-normal statistics are usually adequate to describe the behavior.

The level of scintillation is taken into account in individual pdfs by the scintillation index

$$
\sigma_{I}^{2}=\frac{\left\langle I^{2}\right\rangle-\langle I\rangle^{2}}{\langle I\rangle^{2}}
$$

$I$ is the intensity measured by a point receiver and angle brackets indicate time averages. In practice, the receiver of an OW link will have a finite collection area and will only act as a point receiver if its diameter $D$ is small enough to satisfy $D<<\sqrt{\lambda L}$, where $\lambda$ is the wavelength used and $L$ is the length of the link. If the receiver diameter is larger than that of a point receiver then the level of scintillation is reduced by aperture averaging and the scintillation index in this case is given by Equation (4.2) with $I$ replaced by the received power $P$.

The strength of turbulence is frequently characterized by the Rytov variance. The Rytov variance is obtained by approximating the solution to Maxwell's equations for electromagnetic wave propagation through a random medium under weak turbulence conditions. For a plane wave, the Rytov variance is calculated as

$$
\sigma_{\ln I_{R}}^{2}=\left\langle(\ln I-\langle\ln I\rangle)^{2}\right\rangle=1.23 \cdot C_{n}^{2} k^{7 / 6} L^{11 / 6},
$$

where $C_{n}^{2}$ is the refractive index structure constant and $k$ is the wavevector magnitude of the light. In weak turbulence, when $\sigma_{I}^{2}<0.3$, the Rytov approximation is valid and the scintillation index practically coincides with the Rytov variance. As the turbulence strength increases, however, the Rytov approximation no longer provides a precise solution to Maxwell's equations and the Rytov variance increases without limit. Strong turbulence theory is then required to predict the scintillation index behavior at increasing values of the Rytov variance and it has been shown that the scintillation index saturates at values of the Rytov variance that can range up to 5-6 [158]. Figure 8 show the behavior of the scintillation index, calculated from weak and strong turbulence theory [160], as a function of the Rytov variance.

The aforementioned atmospheric impairments are combined with factors that include (a) the optical power losses in the transmitter and receiver, (b) beam spreading, (c) the Strehl (optical imaging quality) ratio, and (d) pointing errors to calculate link parameters such as the link margin and link availability [164-166]. The link margin is defined as the ratio of the transmitted power to the minimum power that is required to achieve a desired BER at the receiver. Signal fades that exceed the link margin lead to an increased BER for the duration of the fade and extremely deep fades raise the BER to $50 \%$, causing a total loss of data and rendering the link useless. In contrast to other wireless channels, signal fading on the OW channel is relatively slow compared to the data-rate. The duration of a fade might be up to $100 \mathrm{~ms}$ or longer when the clock period, which depends on the data rate, is generally less than $20 \mathrm{~ns}(50 \mathrm{Mb} / \mathrm{s})$. Hence, during a single fade several thousand transmitted bits can be corrupted. The link availability, on the other hand, is measured over a larger timescale and is defined as the percentage of time over a year that the link is available. The link availability is primarily dependent upon the existence of obscurations in the atmosphere. Under heavy obscuration, the atmospheric losses exceed the link margin and, similar to deep fades, the link operates at an increased BER or is unusable for the duration of the obscuration. When link outage resulting from 


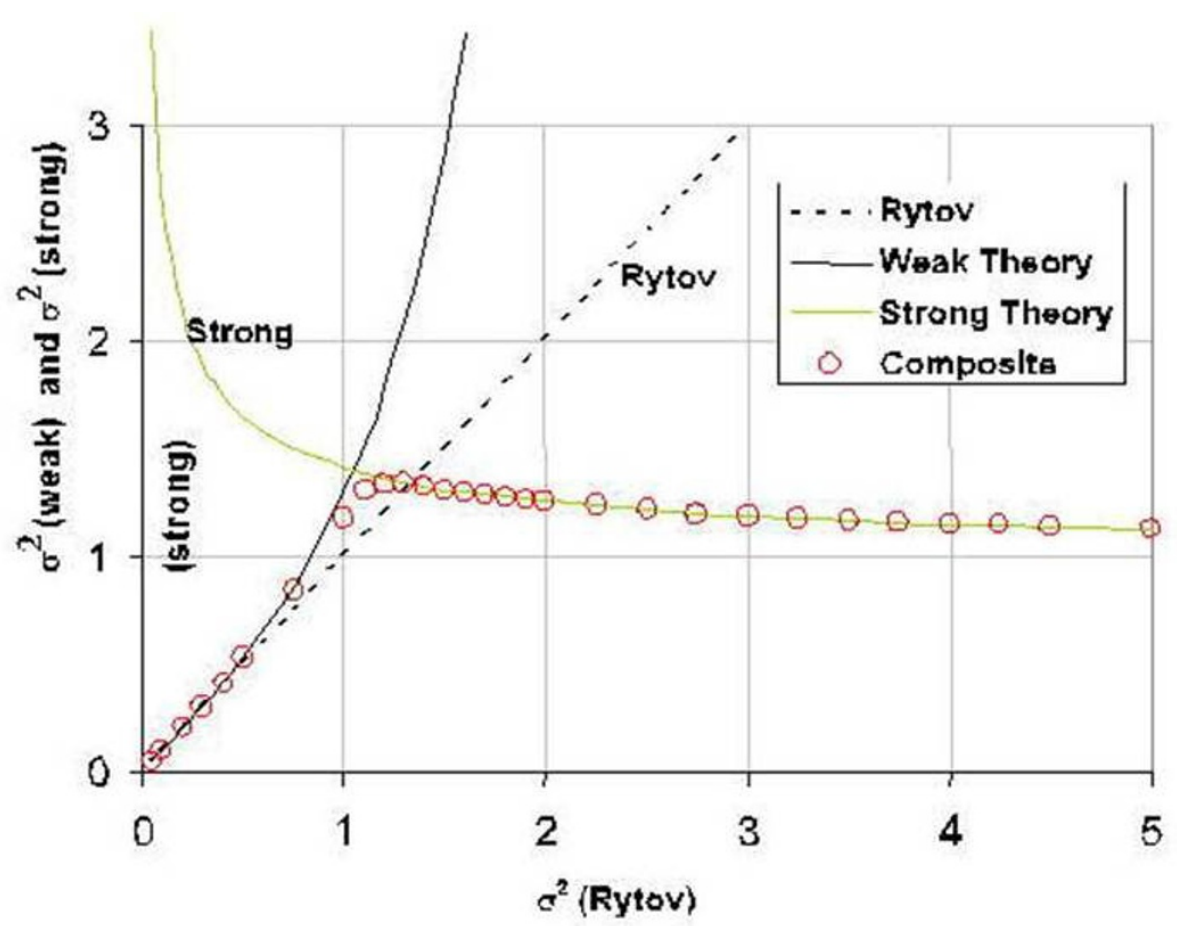

Figure 8 Comparison of log intensity variances calculated using weak and strong turbulence theories [160] for an atmospheric path of $863 \mathrm{~m}$ at $632.8 \mathrm{~nm}$.

obscuration cannot be tolerated the best solution is to operate a hybrid link where the OW link is operated in parallel with a directional RF link in unlicensed regions of C-band (5.8 GHz) or Ku-band. Hybrid OW/RF systems are detailed in the next section.

\subsection{Fade mitigation techniques}

There are four principal methods for mitigating the effects of fading at the physical layer: aperture averaging, diversity, error correction, and adaptive optics.

(1) Aperture averaging: Aperture averaging reduces the scintillation index by using a receiver whose aperture diameter $D$ is larger than the lateral correlation distance on the link $\sqrt{\lambda L}$. Measurements of the effect of the aperture size on scintillation index have been given in [167]. These measurements, made in intermediate strength turbulence, are in reasonable agreement with the weak turbulence formula given in [152]

$$
F=\frac{\sigma_{I}^{2}(D)}{\sigma_{I}^{2}(0)}=\left[1+1.07 \cdot\left(\frac{k D^{2}}{4 L}\right)\right]^{-1}
$$

which describes the reduction $F$ in the scintillation index for a receiver of diameter $D$ compared to a point receiver and holds for plane waves with a small inner scale $l_{0}$ satisfying $l_{0}<<\sqrt{L / k}$. The aperture averaging factor does not depend on the exact shape of the aperture and depends primarily on receiver aperture area [168].

(2) Diversity techniques: Diversity techniques use multiple identical transmissions from sources to receivers $[169,170]$. The multiple transmissions take uncorrelated paths and as a result the effective level of scintillation and the probability of a fade can be reduced. The most common diversity approach uses multiple transmitter and receiver apertures (MIMO), which are spaced further apart than the lateral correlation distance on the link [171-173]. If $n$ independent apertures are used and if the received signals are logically combined then the probability of a fade of a given depth is reduced to prob $\left(P \leq P_{\min }\right)^{n}$, with $P_{\min }$ being the minimum power at each receiver aperture. For a fair comparison of the diversity approach the probability should be evaluated for the same total power transmitted, which means that the fade reduction is really $\operatorname{frac}\left(\frac{P_{\text {Total }}}{n} \leq P_{\min }\right)^{n}$, where $\operatorname{frac}(x)$ denotes the fractional part of $x$.

An alternative approach to spatial diversity is to use time-delayed diversity (TDD), described in $[174,175]$. In this approach, $n$ total transmissions of the same data take place between a single transmitter and a single receiver. The replicated transmissions are delayed by an offset $\tau$ which is longer than expected fade duration. The replicas are re-synchronized, buffered and logically 
combined at the receiver. If the separate transmissions of data are truly independent then the BER is reduced from its single channel value of BER to $\mathrm{BER}^{n}$. In practice, the exact reduction in BER must be calculated from the joint pdf for the signal received at different delay times, which requires knowledge of the temporal covariance matrix. Experimental measurements [175] have shown that in weak turbulence a delay of about 20 ms provides decorrelation between successive transmissions. The required decorrelation time becomes shorter as turbulence gets stronger, which is precisely when diversity approaches of all types become most valuable.

(3) Error correction: In some scenarios and especially for airborne terminals, size, power and weight constraints may not allow for multiple transmissions and therefore diversity cannot be implemented. Additionally, diversity systems can get very complex because of issues such as optics alignment, signal synchronization, interference effects, and others. Error correction is a simpler fade mitigation technique that has been shown to give satisfactory results [176] on SISO (single input single output) channels. Forward-error-correction (FEC), in particular, adds additional bit patterns to each coded packet being transmitted, which allows all transmitted bits to be recovered if some are lost due to fading. For example, in a $(255,223)$ Reed-Solomon code that uses 8bit symbols, there are 255 codeword bytes. Each codeword contains 223 bytes of data and 32 bytes for parity checking. The decoder can correct any 16 symbol errors in the code word: errors up to 16 bytes anywhere in the codeword can be automatically corrected. Still, this method only works well if fades are of short duration. In this example, the fade duration should be limited to 128-bit periods. Unfortunately, the transmission time of a code word in a high data rate OW system is much shorter than even the shortest fades. At $1.25 \mathrm{~Gb} / \mathrm{s}$, which is a commercially available OW data rate, 255 bytes takes only $1.6 \mu \mathrm{s}$ for transmission. As a result, improved coding and interleaving techniques are being investigated for application in higher rate OW systems.

Recent study has suggested that BER improvement can be obtained by the use of low density parity check (LDPC) codes [163]. It is not clear, however, that in this work the temporal characteristics of the strongly fading channel were properly accounted for. The performance of a link-layer LDPC staircase FEC system (these codes are called IRA - Irregular Repeat-Accumulate codes [177]) has also been tested over a Fast-Ethernet OW link [176]. In the presented test scenario the mentioned code was able to overcome all packet losses (11\% of lost data). Another approach is to employ interleaving in parallel with coding. Interleaving provides a temporal diversity by spacing coded symbols by time intervals that are longer than expected fade durations. As a result, a fade only affects a single symbol per word in an interleaved in system and the length of the required code is drastically reduced. Further details about coding and interleaving for OW channels can be found in the specialized literature [177-180].

The drawback of FEC, and especially interleaving, is increased latency. The data have to be buffered at the transmitter and the receiver for at least the duration of the used coding constraint length. A rule of thumb suggests using block lengths that have a constraint length of several times the maximum expected fade duration. The processing time needed for encoding and decoding must also be added to the total processing delay. In general, the overall transmission delay can be expected to exceed $100 \mathrm{~ms}$ and therefore it is not practical to implement FEC-interleaving schemes for delay-critical data. Latency can be alleviated by introducing rate-compatible (or rateless) coding into the error correction scheme [181]. A key aspect of rateless coding is that the receiver is capable of decoding the original information, with high probability, even if only part of the original information is available. To this end, the transmitter creates a sequence of frames based on the original data, and the receiver combines the collected frames and decodes the original data once enough frames have been received.

(4) Adaptive optics: In adaptive optics (AO) a deformable mirror is used to pre-distort a transmitted laser beam so that on its passage through the turbulent atmosphere it arrives at the receiver as an undistorted wave. In most long range OW demonstrations that have been carried out to-date AO has been used to attempt to mitigate the effects of the turbulent channel. Unfortunately, as pointed out by Stotts et al. [164] "the ability of an adaptive optics (AO) system to compensate turbulence along a path is limited by the transmitter and receiver Rayleigh ranges, proportional to the diameter of the optics squared and inverse of the wavelength of light utilized." AO is ineffective when the total range is greater than the sum of the Rayleigh ranges. The Rayleigh range is $R_{R}=0.7 D^{2} / \lambda$, where $D$ is the diameter of the transmitter or receiver aperture, and $\lambda$ is the wavelength. For example with a $1.55-\mu \mathrm{m}$ transmitter, and a $100-\mathrm{mm}$ diameter receiver $R_{R}$ $=4.5 \mathrm{~km}$. An AO receiver can correct the wavefronts that actually arrive at a receiver aperture, for example to couple the light into single mode fiber. But, full reciprocal AO that couples transmitter and receiver is not possible unless the range is sufficiently short, or when the transmitter and receiver are not moving fast laterally with respect to each other. Although AO has had considerable success in optical astronomy, its complexity, cost, and inability to correct for long range effects makes its widespread adoption for $\mathrm{OW}$ problematic. A full discussion of $\mathrm{AO}$ is beyond the scope of this article, but the study of Fried and co-workers [182-187] is particularly important in this context. 


\subsection{Satellite links and high-altitude platforms}

Outdoor OW links are a high-bandwidth and lowpower-consumption alternative in satellite communications [4] and OW links have been successfully demonstrated in satellite-to-satellite, earth-to-satellite and earth-to-moving-vehicle links [188-199]. Satellite systems are the most challenging category of outdoor OW systems, because of the very long distances that the optical beams propagate through vacuum or the atmosphere. Optical links that operate in vacuum (inter-satellite and space communications) are at least tens of thousands of $\mathrm{kms}$ long. Even if the vacuum is considered to be ideal (lossless), the associated path losses that increase with the square of the link distance are enormous, and vary from approximately $150 \mathrm{~dB}$ in satelliteto-satellite communications to approximately $230 \mathrm{~dB}$ in Mars-to-Earth communications. Earth-to-satellite optical links involves shorter distances, up to approximately $36,000 \mathrm{~km}$ for a geostationary orbit, but the total system losses are increased due to scattering and absorption in the atmosphere $[150,152]$. Hence, a key consideration in satellite communications is the received optical power and several techniques have been proposed to mitigate the transmission associated losses. Optical amplification is an appealing candidate [200-203], especially in earthto-satellite uplinks where low power consumption limitations do not apply. Another possible candidate in earth-to-satellite communications is the use of flying vehicles and high-altitude-platforms as beam relays [204-206]. When no amplification or intermediate relays are available, as in deep space communications [207], the losses may not be mitigated and more sophisticated receivers that operate at very low powers (photoncounting) are required [208].

A second important aspect of satellite systems is the selection of suitable modulation formats and coding schemes, depending on the application scope. Power efficiency is critical in satellite communications, since satellites recharge only by means of solar energy. Recently, a 5.6-Gb/s inter-satellite link demonstrated the suitability of binary phase shift keying (BPSK) for satellite OW links [192]. Deep space communications, on the other hand, are plagued by extreme path losses and favor the deployment of modulation formats with high peak-to-average power ratios, which ensure that energy peaks are detected. Multilevel PPM systems have been shown to be suitable for deployment in deep space communications $[150,209]$. Efficient error-correcting coding is also required in deep space communications due to the extremely low powers received, and concatenated convolutional codes have being shown to perform close to the theoretical capacity limit [209]. In earth-to-satellite systems, coherent phase modulation schemes provide high sensitivity and the use of BPSK has been previously reported on a similar $5.6 \mathrm{~Gb} / \mathrm{s} 142 \mathrm{~km}$ OW link [210]. PPM has also been studied under the prism of providing sufficient peak power to penetrate through clouds [211,212]. Error-correcting codes help to mitigate deep atmospheric fades, but there is a high possibility that the OW link will be lost due to adverse weather conditions altogether [213]. In such cases, temporal or spatial diversity is required to maintain a working earthto-satellite optical link $[214,215]$.

A final challenge in satellite communications is beam acquisition, pointing, and tracking. Longer links typically require more accurate and sophisticated acquisition, pointing and tracking systems to maintain an operational OW link, since the slightest error is magnified by the link distance. From a communication perspective, pointing and tracking are not only important for maintaining the optical link, but also contribute to the link power penalty since misalignment caused by pointing and tracking errors result in power fluctuations at the receiver [216-218]. Pointing and tracking errors are caused mainly by (a) satellite vibrations [218], (b) errors in the calculation of the point-ahead angle [4], and (c) beam wander effects [219]. Pointing and tracking errors may be reduced by using advanced automated control methods in the pointing and tracking element [4], while their impact may be dampened by allowing adaptive variations in the transmitted power [218] or a broader optical beam [220]. In earth-to-satellite communications pointing and tracking also suffer from fades in the atmosphere, and it is possible that re-acquisition of the beam is required after a fade, leading to extended capacity loss. Reference [221] discusses how inertial sensors can be utilized to counter-effect beam loss due to atmospheric fading.

\section{Hybrid OW/RF systems}

OW links can support high data rate communications in large unlicensed bandwidths, but their data rates and reliability are severely affected by atmospheric phenomena that include absorption, scattering and atmospheric turbulence [158]. While in clear conditions atmospheric attenuation for an OW link can be as low as a fraction of a few $d B / \mathrm{km}$, heavy snow or fog can increase this attenuation to hundreds of $\mathrm{dBs} / \mathrm{km}$. Atmospheric turbulence, particularly in hot dry climates, causes signal fading that can degrade the link performance significantly. Besides, when the links involve mobility, maintaining accurate pointing and tracking over long distances becomes highly challenging, and link deterioration or complete link failure occurs. A key requirement is therefore to increase the reliability of OW links.

Improving the reliability of OW links, while still exploiting their high data carrying capabilities, is achieved by operating the OW link in conjunction with 
an RF link. Under this scheme of operation, the RF part may be used for link control, maintenance and backup functions that require relatively low line rates, while the broadband OW part transfers the bulk of data. This hybrid operation scheme improves reliability since RF links can be much less sensitive to atmospheric conditions and pointing errors, depending on their frequency of operation. In addition, when the OW links fail due to obscurations or fading, the RF links can act as a backup still providing a significant percentage of the OW system bandwidth. The RF data rate can be increased over a given bandwidth using higher order modulations, for instance quadrature amplitude modulation at the expense of transmitted power and system complexity. Even though RF frequencies have regulation restrictions and large bandwidths for high data rates at desired frequencies are difficult to obtain, unlicensed bands at 2.4, 5 , and $60 \mathrm{GHz}$ are available for the implementation of hybrid OW/RF systems.

The increased reliability of hybrid OW/RF over OWonly links enables them as a low-cost rapidly deployable last-mile communication technology. In last-mile applications, OW/RF can provide connectivity over longer distances within the eye safety limitations of laser radiation. In addition, OW/RF links can be used for both fixed as well as mobile multigigabit backbone networks [222-224]. Such networks may include ship-to-shore, ship-to-ship, ground-to-aircraft, and aircraft-to-aircraft links. The use of hybrid OW/RF systems for mobile robot team applications has been discussed in [225]. Taking advantage of the high bandwidths of OW links and reliability of RF links, such robot teams can transmit real-time video data in applications such as search and rescue operations. The use of hybrid OW/RF systems can also facilitate RF spectrum planning and utilization. If OW/RF hybrid systems are designed to meet a required quality of service then some of the existing RFonly links can be replaced with hybrid links. Hybrid links typically utilize only a fraction of the RF bandwidth in comparison with RF-only links [226]. The released RF spectrum can subsequently be made available for more critical applications.

\subsection{Hybrid OW/RF channel model}

In a hybrid OW/RF system, it is necessary to include both the OW and RF channel impairments in the overall system performance. In general, OW and RF links, depending on the frequency of operation, can be modeled independently to include the weather effects. As a result, separate channel models for the OW and RF links are mostly adequate to predict the hybrid system performance. Most practical OW systems employ intensity modulation with direct detection (IM/DD). The OW channel propagation results in a multiplicative effect on the transmitted optical intensity of the laser source with a channel gain factor. The channel gain includes atmospheric attenuation, pointing error effects and atmospheric turbulence. A detailed discussion of the channel impact on OW only systems has been presented in Section 4. Since the OW link operates with direct line-of-sight (LOS), the RF link too has LOS, and therefore the RF link can be modeled as a Ricean channel and often as an additive white Gaussian noise (AWGN) channel for good channel conditions. However, for longer and high altitude links severe fading in the RF link can occur. One example is [222], where the link operates over a distance of $150 \mathrm{~km}$ at a height of $10,000 \mathrm{ft}$. Both frequency-flat and frequency-selective fading were observed for a system operating in the Xand $\mathrm{Ku}$-band at a data rate of $274 \mathrm{Mb} / \mathrm{s}$.

When higher RF frequencies are considered (such as at millimeter wavelengths) the OW and RF channels may not be truly independent. Higher RF frequency bands support higher data rates, but they are also more affected by atmospheric effects and pointing errors. Consequently, higher RF frequencies are affected by scintillations, and moderate to heavy rain events were reported to affect both OW laser link at $850 \mathrm{~nm}$ and millimeter wave RF [227]. Pointing errors can also affect scintillation statistics for both channels [228]. In such a case, pointing error correlations between the two links cannot be ignored.

\subsection{Hybrid OW/RF link architecture and networking}

The RF link in hybrid OW/RF channels can be used for link acquisition, data transmission control and/or data transfer. Accordingly, hybrid systems are divided into three categories, depending on the usage of the RF link, as follows:

(1) Hybrid OW/RF using RF as the control link: The RF link is generally more reliable than the OW link and less sensitive to pointing. Thus, it can be used to assist in the pointing and acquisition of the OW link. It can also be used to provide packet retransmission requests. Successful RF retransmission requests can significantly improve system performance, as observed in [222] for a multi-Gb/s data rate system.

(2) Hybrid OW/RF with switching and data splitting: Since the OW link is the primary channel, the RF link can be used either as a backup or as a parallel link carrying a fraction of the transmitted data. An experimental $2.9 \mathrm{~km}$ hybrid OW/RF link is described in [229], where the RF link works in a standby mode. The laser link provides connection at $155 \mathrm{Mb} / \mathrm{s}$ and the received signal level is checked every $5 \mathrm{~s}$. When the signal falls below a certain threshold, data transmission switches to the RF link, which operates at $2.4 \mathrm{GHz}$ with a data rate of 11 $\mathrm{Mb} / \mathrm{s}$. Data transmission continues on the RF link until 
an improved signal level is detected and then the transmission switches back to the laser link. Although this hybrid OW/RF experiment is important, this approach under-utilizes resources, as only one link is active at any given time.

When using both OW and RF links concurrently, data is partitioned between the links. In [224], a dynamic load switching algorithm is employed and incremental load shifting from one link to the other is carried out, based on the status of the two links. For this purpose, BER is recorded on both links every minute and the averaging effect over a window is used for switching. A practical coded OW/RF switching scheme using an automatic load distribution between the links is described in [230]. The data packets are encoded using Raptor codes and sent over the two links at their respective rates of $1 \mathrm{~Gb} / \mathrm{s}$ and $96 \mathrm{Mb} / \mathrm{s}$. Both the links employ cyclic redundancy check (CRC) codes and discard corrupted packets. The receiver decodes the entire message when there are a sufficient number of correctly received packets. The transmitter continues sending encoded packets for a given message until it receives a 1-bit feedback message. Although for a given reliability level the data rate of this approach is less due to Raptor code overhead, the average data rate is improved by nearly six times in some cases. The use of Raptor codes for hybrid OW/RF systems is also considered in [231].

(3) Hybrid OW/RF with codeword partitioning: If the transmitted data are encoded for error correction, separate encoding and decoding over OW and RF links do not fully exploit the available diversity. For example, when the OW link deteriorates, a data packet encoded and decoded only over the OW link may still be received in error. If, however, some of the encoded bits are transmitted over a more reliable RF link, there is a higher probability of correctly receiving the packet. This idea has been recently explored by many researchers. In general, as more and more bits are transmitted over a more reliable RF channel, the hybrid link remains operational even with less available OW SNR. This gives rise to trade-offs between OW and RF link parameters.

A non-uniform rate-compatible low density parity check (LDPC) code is proposed in [232] for partitioning data into OW and RF links. The code rate is changed through puncturing of the coded bits. To get insights into the behavior of LDPC codes over hybrid channels, density evolution for hybrid channels is described in [226]. For a given code rate and OW/RF signal-to-noise ratios, the minimum RF data rate needed for the convergence of the decoder algorithm is obtained. This helps in the code requirements and design of hybrid systems. For example, the receiver can ask for an increase or decrease in RF rate in steps. Hybrid channel density evolution analysis for irregular LDPC codes shows that some further improvement in performance can be obtained. The performance improvement is higher for stronger turbulence channels. An experimental testbed verification demonstrating the joint decoding of LDPC codes over the two channels is carried out in [233]. Convolutional and turbo codes for hybrid channels are also investigated in the literature [234,235]. Since these codes require simpler encoders than decoders, they can be useful at transmitters with limited resources. The convolutionally coded bits are partitioned into OW and RF channels using an optimized pattern and interleaved before modulation [234]. This bit-interleaved coded modulation is able to exploit the diversity of the two links. In [235], error analysis for turbo codes in hybrid channels showing trade-offs between RF and OW SNRs is described. Optimal turbo-coded bit allocations for puncturing and RF transmission are analyzed and related algorithms are also presented.

From a networking perspective, hybrid OW/RF networks are required to provide high-rate data connectivity with low end-to-end delay. A multihop network with short length links will provide high availability since the shorter OW links are less vulnerable to outages [224]. However, adding more hybrid nodes is costly and it also increases end-to-end delay. A hybrid wireless network of backbone nodes is considered in [223], where the nodes can be pointed to new nodes if necessary. Topology discovery, dissemination, link restoration, optimal OW/RF configurations and routing are very critical for network operation. These functions are accomplished through cooperation of the RF and OW subsystems. For link restoration, an increase in OW transmit power, bit rate reduction and hybrid link switching were explored. Alternatively, OW beam search along with RF connectivity can be used. If the OW link fails in a switched hybrid system then the connection switches to the RF link and optical beam probing can be continued until the OW link is restored [223]. A detailed OW/RF network analysis, with links using codeword partitioning, is an open research problem.

\section{Conclusions and future directions}

This article has presented an overview of OW technologies, emphasizing their deployment in communication systems. Four distinct OW system categories (personal communication, indoor, outdoor, and hybrid OW/RF) have been discussed and key requirements and major applications areas have been identified for each category. Personal communication OW systems are required to provide high rate connectivity and very short connection establishment times. Both have been implemented in the IrDA-standardized physical layer and protocol stack. The current state of the art has enabled data transmission at $\mathrm{Gb} / \mathrm{s}$ speeds and this technology is currently 
under standardization by the IrDA Giga-IR group. Future standardization efforts include a technology merge of infrared and USB technologies (IrUSB), as well as a comprehensive analysis and optimization of available protocols with the new $\mathrm{Gb} / \mathrm{s}$ speeds in mind.

Indoor OW systems are required to allow for user mobility, which can be quite challenging due to the dispersive nature of the indoor channel. Dispersion mitigation is of critical importance in indoor OW and suitable mitigation techniques, including multi-spot diffusing links and coding, have been presented in this article. The deployment of OW has been limited in the past years, but recently VLCs have emerged as a future highpotential indoor OW technology. VLCs are being actively investigated by the VLCs Consortium and the IEEE 802.15.7 Task Group. The VLCs Consortium has already produced standards for VLCs and is currently cooperating with IrDA to promote OW systems for personal and indoor communications.

Outdoor OW systems are required to provide high data rates and remain functional at link distances that range from hundreds of meters to thousands of $\mathrm{kms}$. Outdoor systems are seriously affected by atmospheric impairments, mainly obscuration and fading. These effects are increasingly deleterious as the link length increases and mitigation techniques like temporal/spatial diversity, coding/interleaving and aperture averaging are required to maintain link operation. At present, $10 \mathrm{~Gb} / \mathrm{s}$ Ethernet compliant outdoor OW systems are commercially available for metro applications from several vendors. In the near future, link speeds are expected to rise, following the bandwidth increase in high-speed optical components that currently operate at 40-100 Gb/s.

Finally, hybrid OW/RF systems have been introduced to augment outdoor OW systems with increased reliability. The inclusion of the RF subsystem facilitates link establishment and operation, enhances error correction and data transfer control, and provides a backup link in the event of a failure. Hybrid systems are currently under research and researchers aim to identify which cooperation schemes between the OW and RF links provide the most reliable overall system operation.

\footnotetext{
Author details

${ }^{1}$ Klipsch School of Electrical and Computer Engineering, New Mexico State University, Las Cruces, NM, USA ${ }^{2}$ Department of Telecommunications Science and Technology, University of Peloponnese, Tripoli, Greece ${ }^{3}$ Department of Electrical and Computer Engineering, University of Maryland, College Park, MD, USA ${ }^{4}$ Department of Electrical and Computer Engineering, McMaster University, Hamilton, ON, Canada ${ }^{5}$ Department of Telecommunications Science and Technology, University of Peloponnese, Tripoli, Greece
}

\section{Competing interests}

The authors declare that they have no competing interests.
Received: 6 June 2011 Accepted: 7 March 2012 Published: 7 March 2012

\section{References}

1. F Gfeller, U Bapst, Wireless in-house data communication via diffuse infrared radiation. Proc IEEE. 67(11), 1474-1486 (1979)

2. Infrared Data Association (IrDA) http://www.irda.org

3. Visible Light Communications Consortium http://www.vlcc.net

4. WW Chan, Optical satellite networks. IEEE/OSA J Lightw Technol. 21(11), 2811-2827 (2003)

5. IEEE Standard for Wireless LAN Medium Access Control (MAC) and Physical Layer (PHY) Specifications (1997)

6. IEEE 802.15 Working Group for WPAN http://www.ieee802.org/15/

7. IrDA, Advanced Infrared (Alr) MAC Draft Protocol Specification-Version 1.0 Infrared Data Association. (1999)

8. IrDA, Advanced Infrared Logical Link Control (AlrLC) Specification-Version 0.1, Infrared Data Association. (1999)

9. IrDA, Advanced Infrared Physical Layer Specification (Alr-PHY) -Version 1.0, Infrared Data Association. (1998)

10. IrDA, Point and Shoot Profile-Version 1.1, Infrared Data Association. (2000)

11. IrDA, Physical Layer Specification-Version 1.4, Infrared Data Association. (2001)

12. IrDA, Serial Infrared Physical Layer Specification-Giga-IR Addition Version 1.0, Infrared Data Association. (2009)

13. IEC 60825-1, Safety of Laser Products-Part 1: equipment classification, requirements, and user's guide, edition 1.2. International Electrotechnical Commission (2001)

14. IrDA, Serial Infrared Link Access Protocol (IrLAP) -Version 1.1, Infrared Data Association. (1996)

15. IrDA, Link Management Protocol (IrLMP)-Version 1.1, Infrared Data Association. (1996)

16. IrDA, Tiny TP: a flow-control mechanism for use with IrLMP-Version 1.1, Infrared Data Association. (1996)

17. IrDA, Object Exchange Protocol (OBEX $\left.{ }^{\mathrm{TM}}\right)$-Version 1.5, Infrared Data Association. (2009)

18. IrDA, Link Access Protocol Specification for Giga-IR Addition-Version 1.0 Infrared Data Association. (2009)

19. IrDA, IrLAP Fast Connect (Application Note)-Version 1.0, Infrared Data Association. (2002)

20. IrDA, Minimal IrDA Protocol Implementation (IrDA Lite)-Version 1.0, Infrared Data Association. (1996)

21. IrDA, IrSimple (Infrared Simple) Profile-Version 1.01, Infrared Data Association. (2007)

22. IrDA, Serial Infrared Sequence Management Protocol for IrSimple-Version 1.0, Infrared Data Association. (2005)

23. IrDA, Infrared Financial Messaging $\left(\operatorname{IrFM} M^{\top M}\right)$ Point and Pay Profile-Version 1.0, Infrared Data Association. (2002)

24. IrDA, 'IrCOMM': Serial and Parallel Port Emulation over IR (Wire Replacement)-Version 1.0, Infrared Data Association. (1995)

25. IrDA, Infrared Universal Bus Specification-Version 0.11, Infrared Data Association. (2010)

26. AC Boucouvalas, Indoor ambient light noise and its effect on wireless optical links. IEE Proc Optoelectron. 143(6), 334-338 (1996)

27. J Kahn, J Barry, Wireless infrared communications. Proc IEEE. 85(2), 265-298 (1997)

28. JM Kahn, WJ Krause, JB Carruthers, Experimental characterization of nondirected indoor infrared channels. IEEE Trans Commun. 43(234), 1613-1623 (1995)

29. R Pérez-Jiménez, JA Rabadán, FJ López-Hernández, Filtered modulation schemes for short distance infrared wireless communications. IEEE Trans Consumer Electron. 46(2), 275-282 (2000)

30. RT Valadas, AR Tavares, AM de Oliveira Duarte, AC Moreira, CT Lomba, The infrared physical layer of the IEEE 802.11 Standard for wireless local area networks. IEEE Commun Mag. 36(12), 107-112 (1998)

31. D Shiu, JM Kahn, Differential pulse position modulation for power-efficient optical communication. IEEE Trans Commun. 47(8), 1201-1210 (1999)

32. Z Ghassemlooy, AR Hayes, Digital pulse interval modulation for IR communication systems-a review. Int J Commun Sys. 13(7-8), 519-536 (2000)

33. W Hirt, M Hassner, $N$ Heise, IrDA-VFIr $(16 \mathrm{Mb} / \mathrm{s})$ : modulation code and system design. IEEE Personal Commun. 8(1), 58-71 (2001) 
34. AX Widmer, PA Franaszek, A DC-balanced, partitioned-block, 8B/10B transmission code. IBM J Res Dev. 27(5), 440-451 (1983)

35. AM Street, K Samaras, DC O'Brien, DJ Edwards, Closed form expressions for baseline wander effects in wireless IR applications. IEE Electron Lett. 33(12), 1060-1062 (1997)

36. Z Ghassemlooy, Investigation of the baseline wander effect on indoor optical wireless system employing digital pulse interval modulation. IET Commun. 2(1), 53-60 (2008)

37. JB Carruthers, JM Kahn, Multiple-subcarrier modulation for non-directed wireless infrared communication. IEEE J Sel Areas Commun. 14(3), 538-546 (1996)

38. R You, JM Kahn, Average power reduction techniques for multiplesubcarrier intensity-modulated optical signals. IEEE Trans Commun. 49(12), 2164-2171 (2001)

39. O Gonzalez, R Perez-Jimenez, S Rodriguez, J Rabadan, A Ayala, OFDM over indoor wireless optical channel. IEE Proc Optoelectron. 152(4), 199-204 (2005)

40. R Mesleh, H Elgala, H Haas, On the performance of different OFDM based optical wireless communication systems. OSA J Opt Commun Netw. 3, 620-628 (2011)

41. J Armstrong, OFDM for optical communications. IEEE/OSA J Lightw Technol. 27(3), 189-2041 (2009)

42. $P$ Chatzimisios, AC Boucouvalas, Packet delay analysis of the advanced infrared (Alr) CSMA/CA MAC protocol in optical wireless LANs. Int J Commun Syst. 18(3), 307-331 (2005)

43. V Vitsas, AC Boucouvalas, Performance analysis of the advanced infrared (Alr) CSMA/CA MAC protocol for wireless LANs. Wirel Netw. 9(5), 495-507 (2003)

44. P Barker, $V$ Vitsas, AC Boucouvalas, Simulation analysis of advanced infrared (Alr) MAC wireless communications protocol. IEE Proc Circ Dev Syst. 149(3), 193-197 (2002)

45. G Bianchi, Performance analysis of the IEEE 802.11 distributed coordination function. IEEE J Sel Areas Commun. 18(3), 535-547 (2000)

46. BP Crow, I Widjaja, LG Kim, PT Sakai, IEEE 802.11 wireless local area networks. IEEE Commun Mag. 35(9), 116-126 (1997)

47. P Huang, P Chatzimisios, A Boucouvalas, Optimizing IrDA throughput by including processing time with physical layer consideration. OSA J Opt Netw. 4, 323-331 (2005)

48. V Vitsas, AC Boucouvalas, Optimization of IrDA IrLAP link access protocol. IEEE Trans Wirel Commun. 2(5), 926-938 (2003)

49. AC Boucouvalas, Pi Huang, Modelling and optimising TinyTP over IrDA stacks. EURASIP J Wirel Commun Netw. 2005(1), 45-56 (2005)

50. AC Boucouvalas, Pi Huang, OBEX over IrDA: performance analysis and optimization by considering multiple applications. IEEE/ACM Trans Netw. 14(6), 1292-1301 (2006)

51. CT Deccio, J Ekstrom, DR Partridge, KB Tew, CD Knutson, A study of the suitability of IrOBEX for high-speed exchange of large data objects, in Proc IEEE GLOBECOM, vol. 5. San Francisco, pp. 2664-2668 (2003)

52. AM Shah, SS Ara, G Kitazumi, M Matsumoto, IrSimple Modeling and Performance Evaluation for High-Speed Infrared Communications, in Proc IEEE GLOBECOM, San Francisco, pp. 1-6 (2006)

53. IrDA, Burst Data Protocol (IrBurst)-Version 1.0, Infrared Data Association. (2004)

54. MS Alam, SA Shawkat, K Gontaro, M Mitsuji, IrBurst modeling and performance evaluation for large data block exchange over high-speed IrDA links. IEICE Trans Commun. 91(1), 274-285 (2008)

55. P Huang, A Boucouvalas, IrBurst modelling and performance analysis in the presence of transmission errors. Wirel Personal Commun. 41(1), 111-125 (2007)

56. P Huang, A Boucouvalas, Future personal "e-payment": IRFM. IEEE Wirel Commun. 13(1), 60-66 (2006)

57. T-S Chu, M Gans, High speed infrared local wireless communication. IEEE Commun Mag. 25(8), 4-10 (1987)

58. DJT Heatley, DR Wisely, I Neild, P Cochrane, Optical wireless: the story so far. IEEE Commun Mag. 36(12), 72-74 (1998). 79-82

59. G Pang, $\mathrm{T}$ Kwan, H Liu, C-H Chan, LED wireless. IEEE Ind Appl Mag. 8(1), 21-28 (2002)

60. S Hranilovic, Wireless Optical Communication Systems, (Springer, New York, NY, 2004)

61. JR Barry, JM Kahn, Link design for nondirected wireless infrared communications. OSA Appl Opt. 34, 3764-3776 (1995)
62. K Wang, A Nirmalathas, C Lim, E Skafidas, $4 \times 12.5 \mathrm{~Gb} / \mathrm{s}$ WDM optical wireless communication system for indoor applications. IEEE/OSA J Lightw Technol. 29(13), 1988-1996 (2011)

63. MR Pakravan, E Simova, M Kavehrad, Holographic diffusers for indoor infrared communication systems. Int J Wirel Inf Netw. 4(4), 259-274 (1997)

64. H Elgala, R Mesleh, H Haas, Indoor broadcasting via white LEDs and OFDM. IEEE Trans Consumer Electron. 55(3), 1127-1134 (2009)

65. V Jungnickel, A Forck, T Haustein, U Kruger, V Pohl, C von Helmolt, Electronic tracking for wireless infrared communications. IEEE Trans Wirel Commun. 2(5), 989-999 (2003)

66. DV Plant, AG Kirk, Optical interconnects at the chip and board level: challenges and solutions. Proc IEEE. 88(6), 806-818 (2000)

67. G Kim, X Han, R Chen, An 8-Gb/s optical backplane bus based on microchannel interconnects: design, fabrication, and performance measurements. IEEE/OSA J Lightw Technol. 18(11), 1477-1486 (2000)

68. MG Bevan, MAG Darrin, SC Walts, W Schneider, CS Mills, RF Conde, Freespace optical data bus for spacecraft, in Proceedings of the $3 \mathrm{rd}$ annual Earth Science Technology Conference, Maryland, USA (June 2003)

69. DC O'Brien, GE Faulkner, EB Zyambo, K Jim, DJ Edwards, P Stavrinou, G Parry, J Bellon, MJ Sibley, VA Lalithambika, VM Joyner, RJ Samsudin, DM Holburn, RJ Mears, Integrated transceivers for optical wireless communications. IEEE J Sel Topics Quantum Electron. 11(1), 173-183 (2005)

70. D Miller, Device requirements for optical interconnects to silicon chips. Proc IEEE. 97(7), 1166-1185 (2009)

71. RM Shelby, JA Hoffnagle, GW Burr, CM Jefferson, M-P Bernal, H Coufal, RK Grygier, H Gunther, RM Macfarlane, GT Sincerbox, Pixel-matched holographic data storage with megabit pages. OSA Opt Lett. 22(19), 1509-1511 (1997)

72. GW Burr, J Ashley, H Coufal, RK Grygier, JA Hoffnagle, CM Jefferson, B Marcus, Modulation coding for pixel-matched holographic data storage. OSA Opt Lett. 22(9), 639-641 (1997)

73. E Bisaillon, DF Brosseau, T Yamamoto, M Mony, E Bernier, D Goodwill, DV Plant, AG Kirk, Free-space optical link with spatial redundancy for misalignment tolerance. IEEE Photon Technol Lett. 14(2), 242-244 (2002)

74. S Hranilovic, FR Kschischang, Short-range wireless optical communication using pixelated transmitters and imaging receivers, in Proceedings of the IEEE International Conference on Communications, vol. 2. Paris, France, pp. 891-895 (20-24 June 2004)

75. S Hranilovic, FR Kschischang, A pixelated MIMO wireless optical communication system. IEEE J Sel Topics Quantum Electron. 12(4), 859-874 (2006)

76. R Mesleh, H Elgala, H Haas, Optical spatial modulation. OSA J Opt Commun Netw. 3(3), 234-244 (2011)

77. D Takase, T Ohtsuki, Spatial multiplexing in optical wireless MIMO communications over indoor environment. IEICE Trans Commun. E89-B(4), 1364-1370 (2006)

78. R Villan, S Voloshynovskiy, O Koval, T Pun, Multilevel 2-D bar codes: toward high-capacity storage modules for multimedia security and management. IEEE Trans Inf Foren Sec. 1(4), 405-420 (2006)

79. MDA Mohamed, S Hranilovic, Two-dimensional binary halftoned optical intensity channels. IET Commun. 2(1), 11-17 (2008)

80. $\mathrm{PH}$ Siegel, Information-theoretic limits of two-dimensional optical recording channels, in Proceedings of the IEEE Optical Data Storage Topical Meeting, Montreal, QC, Canada, pp. 165-167 (23-26 April 2006)

81. L Huang, G Mathew, TC Chong, Channel modeling and target design for two-dimensional optical storage systems. IEEE Trans Mag. 41(8), 2414-2424 (2005)

82. J Joseph, DA Waldman, Homogenized Fourier transform holographic data storage using phase spatial light modulators and methods for recovery of data from the phase image. Appl Opt. 45, 6374-6380 (2006)

83. A Pu, D Psaltis, High-density recording in photopolymer-based holographic three-dimensional disks. OSA Appl Opt. 35(14), 2389-2398 (1996)

84. D Takase, T Ohtsuki, Optical wireless MIMO communications (OMIMO), in IEEE Global Telecom Conf, vol. 2. Dallas, pp. 928-932 (November 2004)

85. J Faucher, MB Venditti, DV Plant, Application of parallel forward-error correction in two-dimensional optical-data links. IEEE/OSA J Lightw Technol. 21(2), 466-475 (2003)

86. E Ohbuchi, H Hanaizumi, LA Hock, Barcode readers using the camera device in mobile phones, in Int Conference on Cyberworlds, Tokyo, Japan, pp. 260-265 (November 2004) 
87. JM Kahn, JR Barry, MD Audeh, JB Carruthers, WJ Krause, GW Marsh, Nondirected infrared links for high-capacity wireless LANs. IEEE Personal Commun. 1(2), 12-25 (1994)

88. M Kotzin, A van den Heuvel, A duplex infra-red system for inbuilding communications, in Proceedings of the 36th IEEE Vehicular Technology Conference, vol. 36. Dallas, Texas, pp. 179-185 (May 1986)

89. JR Barry, JM Kahn, WJ Krause, EA Lee, DG Messerschmitt, Simulation of multipath impulse response for indoor wireless optical channels. IEEE J Sel Areas Commun. 11(3), 367-379 (1993)

90. YA Alqudah, M Kavehrad, MIMO characterization of indoor wireless optical link using a diffuse-transmission configuration. IEEE Trans Commun. 51(9), 1554-1560 (2003)

91. H Hashemi, G Yun, M Kavehrad, F Behbahani, PA Galko, Indoor propagation measurements at infrared frequencies for wireless local area networks applications. IEEE Trans Veh Technol. 43(3), 562-576 (1994)

92. MR Pakravan, M Kavehrad, Indoor wireless infrared channel characterization by measurements. IEEE Trans Veh Technol. 50(4), 1053-1073 (2001)

93. JB Carruthers, JM Kahn, Modeling of nondirected wireless infrared channels. IEEE Trans Commun. 45(10), 1260-1268 (1997)

94. V Jungnickel, V Pohl, S Nonnig, C von Helmolt, A physical model of the wireless infrared communication channel. IEEE J Sel Areas Commun. 20(3), 631-640 (2002)

95. H-S Lee, A photon modeling method for the characterization of indoor optical wireless communication. Prog Electromagn Res PIER. 92, 121-136 (2009)

96. FJ Lopez-Hernandez, R Perez-Jimenez, A Santamaria, Ray-tracing algorithms for fast calculation of the channel impulse response on diffuse IR wireless indoor channels. Opt Eng. 39(10), 2775-2780 (2000)

97. O Gonzalez, S Rodriguez, R Perez-Jimenez, BR Mendoza, A Ayala, Error analysis of the simulated impulse response on indoor wireless optical channels using a Monte Carlo-based ray-tracing algorithm. IEEE Trans Commun. 53(1), 124-130 (2005)

98. O González, S Rodríguez, R Pérez-Jiménez, BR Mendoza, A Ayala, Comparison of Monte Carlo ray-tracing and photon-tracing methods for calculation of the impulse response on indoor wireless optical channels. OSA Opt Express. 19, 1997-2005 (2011)

99. Y Cocheril, R Vauzelle, A new ray-tracing based wave propagation model including rough surfaces scattering. Prog Electromagn Res PIER. 75, 357-381 (2007)

100. MD Audeh, JM Kahn, JR Barry, Performance of pulse position modulation on measured non-directed indoor infrared channels. IEEE Trans Commun. 44(6), 654-659 (1996)

101. MD Audeh, JM Kahn, JR Barry, Decision-feedback equalization of pulseposition modulation on measured nondirected indoor infrared channels. IEEE Trans Commun. 47(4), 500-503 (1999)

102. DC Lee, JM Kahn, MD Audeh, Trellis-coded pulse position modulation for indoor wireless infrared communications. IEEE Trans Commun. 45(9), 1080-1087 (1997)

103. DC Lee, JM Kahn, Coding and equalization for PPM on wireless infrared channels. IEEE Trans Commun. 47(2), 255-260 (1999)

104. F Gfeller, W Hirt, Advanced infrared (Alr): physical layer for reliable transmission and medium access, in Proceedings of the International Zurich Seminar on Broadband Communications, Zurich, Switzerland, pp. 77-84 (1517 February 2000)

105. D Lee, J Kahn, Experimental 25-Mb/s wireless infrared link using 4-PPM with scalar decision-feedback equalization, in Proceedings of the IEEE International Conference on Communications, vol. 1. Atlanta, GA, USA, pp. 26-30 (June 1998)

106. GW Marsh, JM Kahn, 50-Mb/s diffuse infrared free-space link using on-off keying with decision-feedback equalization. IEEE Photon Technol Lett. 6(10), 1268-1270 (1994)

107. GW Marsh, JM Kahn, Performance evaluation of experimental 50-Mb/s diffuse infrared wireless link using on-off keying with decision-feedback equalization. IEEE Trans Commun. 44(11), 1496-1504 (1996)

108. G Yun, M Kavehrad, Indoor infrared wireless communications using spot diffusing and fly-eye receivers. Can J Electron Comput E. 18(4), 151-157 (1993)

109. FE Alsaadi, JMH Elmirghani, Mobile multigigabit indoor optical wireless systems employing multibeam power adaptation and imaging diversity receivers. OSA J Opt Commun Netw. 3, 27-39 (2011)
110. FE Alsaadi, JMH Elmirghani, High-speed spot diffusing mobile optical wireless system employing beam angle and power adaptation and imaging receivers. IEEE/OSA J Lightw Technol. 28(16), 2191-2206 (2010)

111. ST Jivkova, BA Hristov, M Kavehrad, Power-efficient multispot-diffuse multiple-input-multiple-output approach to broad-band optical wireless communications. IEEE Trans Veh Technol. 53(3), 882-889 (2004)

112. ST Jivkova, M Kavehrad, Multispot diffusing configuration for wireless infrared access. IEEE Trans Commun. 48(6), 970-978 (2000)

113. ST Jivkova, M Kavehrad, Holographic optical receiver front end for wireless infrared indoor communications. OSA Appl Opt. 40, 2828-2835 (2001)

114. JM Kahn, R You, P Djahani, AG Weisbin, BK Teik, A Tang, Imaging diversity receivers for high-speed infrared wireless communication. IEEE Commun Mag Vol. 36, 88-94 (1998)

115. J Carruthers, JM Kahn, Angle diversity for nondirected wireless infrared communication. IEEE Trans Commun. 48(6), 960-969 (2000)

116. S Jivkova, M Kavehrad, Receiver designs and channel characterization for multi-spot high-bit-rate wireless infrared communications. IEEE Trans Commun. 49(12), 2145-2153 (2001)

117. M Kavehrad, S Jivkova, Indoor broadband optical wireless communications: optical subsystems design and their impact on channel characteristics. IEEE Wirel Commun Mag Vol. 10, 30-35 (2003)

118. AG Al-Ghamdi, JMH Elmirghani, Line strip spot-diffusing transmitter configuration for optical wireless systems influenced by background noise and multipath dispersion. IEEE Trans Commun. 52(1), 37-45 (2004)

119. AG Al-Ghamdi, JMH Elmirghani, Multiple spot diffusing geometries for indoor optical wireless communication systems. Int J Commun Syst. 16, 909-922 (2003)

120. V Jungnickel, C von Helmolt, U Kruger, Broadband wireless infrared LAN architecture compatible with ethernet protocol. Electron Lett. 34(25), 2371-2372 (1998)

121. R Narasimhan, MD Audeh, JM Kahn, Effect of electronic-ballast fluorescent lighting on wireless infrared links. IEE Proc Optoelectron. 143(6), 347-354 (1996)

122. AJC Moreira, RT Valadas, AM de Olveira Duarte, Optical interference produced by artificial light. Wirel Netw. 3(2), 131-140 (1997)

123. XN Fernando, S Krishnan, H Sun, KM Kamyar, Adaptive denoising at infrared wireless receivers. Proc SPIE. 5074, 199-207 (2003)

124. S Rajbhandari, Z Ghassemlooy, M Angelova, Effective denoising and adaptive equalization of indoor optical wireless channel with artificial light using the discrete wavelet transform and artificial neural network. IEEE/OSA J Lightw Technol. 27(20), 4493-4500 (2009)

125. S Rajbhandari, Z Ghassemlooy, M Angelova, Wavelet-artificial neural network receiver for indoor optical wireless communications. IEEE/OSA J Lightw Technol. 29(17), 2651-2659 (2011)

126. KK Wong, T O'Farrell, Spread spectrum techniques for indoor wireless IR communications. IEEE Wirel Commun. 10(2), 54-63 (2003)

127. T Komine, M Nakagawa, Fundamental analysis for visible-light communication system using LED lights. IEEE Trans Consumer Electron. 50(1), 100-107 (2004)

128. D O'Brien, M Katz, Optical wireless communications within fourthgeneration wireless systems [invited]. OSA J Opt Netw. 4(6), 312-322 (2005)

129. Japan Electronics and Information Technology Industries Association http:// www.jeita.or.jp

130. OMEGA, the Home Gigabit Access Project http://www.ict-omega.eu/

131. The OMEGA Project Flyer [Online]. Available: http://www.ict-omega.eu/ fileadmin/documents/flyers/OMEGA_Project_Flyer.pdf

132. Future networks, Projects Portfolio, Seventh EU Framework Programme for Research and Technological Development, Information Society and Media, European Commission (March 2008)

133. IEEE 802.15 WPAN-Visible Light Communication Interest Group (IGvlc) http://ieee802.org/15/pub/IGvlc.html

134. H Sugiyama, S Haruyama, M Nakagawa, Experimental investigation of modulation method for visible-light communications. IEICE Trans Commun. E89-B(12), 3393-3400 (2006)

135. H Elgala, R Mesleh, H Haas, B Pricope, OFDM visible light wireless communication based on white LEDs, in Proc IEEE Vehicular Technol Conf, vol. 4212879. (Dublin, Ireland, 2007), pp. 2185-2189

136. J Grubor, S Randel, K-D Langer, JW Walewski, Broadband information broadcasting using LED-based interior lighting. IEEE/OSA J Lightw Technol. 26(24), 3883-3892 (2008) 
137. L Zeng, DC O’Brien, HL Minh, GE Faulkner, K Lee, D Jung, Y Oh, T Won, High data rate multiple input multiple output (MIMO) optical wireless communications using white led lighting. IEEE J Sel Areas Commun. 27(9), 1654-1662 (2009)

138. JK Kwon, Inverse source coding for dimming in visible light communications using NRZ-OOK on reliable links. IEEE Photon Technol Lett. 22(19), 1455-1457 (2010)

139. K Sunghwan, J Sung-Yoon, Novel FEC coding scheme for dimmable visible light communication based on the modified Reed-Muller codes. IEEE Photon Technol Lett. 23(20), 1514-1516 (2011)

140. T Komine, JH Lee, S Haruyama, M Nakagawa, Adaptive equalization system for visible light wireless communication utilizing multiple white led lighting equipment. IEEE Trans Wirel Commun. 8(6), 2892-2900 (2009)

141. HL Minh, D O'Brien, G Faulkner, L Zeng, K Lee, D Jung, Y Oh, 80 Mbit/s visible light communications using pre-equalized white LED, in Proc Eur Conf Opt Commun no. P.6.06, Brussels, Belgium, pp. 1-2 (2008)

142. HL Minh, D O'Brien, G Faulkner, L Zeng, K Lee, D Jung, Y Oh, ET Won, 100$\mathrm{Mb} / \mathrm{s}$ NRZ visible light communications using a postequalized white LED. IEEE Photon Technol Lett. 21(15), 1063-1065 (2009)

143. J Grubor, SCJ Lee, K-D Langer, T Koonen, JW Walewski, Wireless high-speed data transmission with phosphorescent white-light LEDs, in Proc Eur Conf Opt Commun, PD3.6, Berlin, Germany, pp. 1-2 (2007)

144. J Vučić, C Kottke, S Nerreter, K-D Langer, JW Walewski, 513 Mbit/s visible light communications link based on DMT-modulation of a white LED. IEEE/ OSA J Lightw Technol. 28(24), 3512-3518 (2010)

145. J Vučić, C Kottke, S Nerreter, A Büttner, K-D Langer, JW Walewski, White light wireless transmission at $200+\mathrm{Mb} / \mathrm{s}$ net data rate by use of discretemultitone modulation. IEEE Photon Technol Lett. 21(20), 1511-1513 (2009)

146. K Lee, H Park, Modulations for visible light communications with dimming control. IEEE Photon Technol Lett. 23(16), 1136-1138 (2011)

147. IEEE 802.15.7 VLC PHY/MAC Proposal https://mentor.ieee.org/802.15/dcn/09/ 15-09-0733-00-0007-vlc-phy-mac-proposal-samsung-etri.pdf

148. G Ntogari, T Kamalakis, JW Walewski, T Sphicopoulos, Combining illumination dimming based on pulse-width modulation with visible-light communications based on discrete multitone. OSA J Opt Commun Netw. 3 , 56-65 (2011)

149. Q Liu, C Qiao, G Mitchell, S Stanton, Optical wireless communication networks for first- and last-mile broadband access [Invited]. OSA J Opt Netw. 4, 807-828 (2005)

150. WWS Chan, Free-space optical communications. IEEE/OSA J Lightw Technol. 24(12), 4750-4762 (2006)

151. A Zilberman, NS Kopeika, Laser beam wander in the atmosphere: implications for optical turbulence vertical profile sensing with imaging LIDAR. J Appl Remote Sens. 2(023540) (2008)

152. LC Andrews, RL Phillips, Laser Beam Propagation Through Random Media, 2nd edn. (SPIE Press, Bellingham, WA, 2005)

153. VI Tatarski, Wave Propagation in a Turbulent Medium (McGraw-Hill, New York, 1961). (Reprinted by Dover, New York, 1967)

154. LA Chernov, Wave Propagation in a Random Medium (McGraw-Hill, New York, 1960). (Reprinted by Dover, New York, 1967)

155. AK Ishimaru, in Wave Propagation and Scattering in Random Media, vol. I and II. (Academic Press, New York, 1978). Vol. II Chapters 16-20 are especially relevant

156. JW Strohbehn, Laser Beam Propagation Through the Atmosphere (SpringerVerlag, New York, 1978)

157. VE Zuev, Laser Beams in the Atmosphere (Consultants Bureau, New York, 1982)

158. LC Andrews, RL Phillips, CY Hopen, Laser Beam Scintillation with Applications (SPIE Press, Bellingham, WA, 2001)

159. RS Lawrence, JW Strohbehn, A survey of clear-air propagation effects relevant to optical communications. Proc IEEE. 58, 1523-1545 (1970)

160. RW Fante, Electromagnetic beam propagation in turbulent media. Proc IEEE. 63, 1669-1692 (1975)

161. HT Yura, WG McKinley, Optical scintillation statistics for IR ground-to-space laser communication systems. OSA Appl Opt. 22, 3353-3358 (1983)

162. MA Al-Habash, LC Andrews, RL Phillips, Mathematical model for the irradiance probability density function of a laser beam propagating through turbulent media. Opt Eng. 40(8), 1554-1562 (2001)

163. JA Anguita, IB Djordjevic, MA Neifeld, BA Vasic, Shannon capacities and error-correction codes for optical atmospheric turbulence channels. OSA J Opt Netw. 4(9), 586-601 (2005)
164. LB Stotts, P Kolodzy, A Pike, B Graves, D Daugherty, J Douglas, Free space optical communication link budget estimation. OSA Appl Opt. 49(28), 5333-5343 (2010)

165. S Arnon, Effects of atmospheric turbulence and building sway on optical wireless-communication systems. OSA Opt Lett. 28, 129-131 (2003)

166. D Kedar, S Arnon, Optical wireless communication through fog in the presence of pointing errors. OSA Appl Opt. 42, 4946-4954 (2003)

167. H Yuksel, S Milner, CC Davis, Aperture averaging for optimizing receiver design and system performance on free-space optical communication links. OSA J Opt Netw. 4(8), 462-475 (2005)

168. H Yuksel, CC Davis, Aperture averaging analysis and aperture shape invariance of received scintillation in free space optical communication links, in Proc SPIE Free-Space Laser Commun VI, vol. 6304. (San Diego, 2006)

169. SG Wilson, M Brandt-Pearce, Q Cao, M Baedke, Optical repetition MIMO transmission with multi-pulse PPM. IEEE J Sel Areas Commun. 23(9), 1901-1910 (2005)

170. SM Navidpour, M Uysal, M Kavehrad, BER performance of free-space optical transmission with spatial diversity. IEEE Trans Wirel Commun. 6(8), 2813-2819 (2007)

171. A Goldsmith, SA Jafar, N Jindal, S Vishwanath, Capacity limits of MIMO channels. IEEE J Sel Areas Commun. 21(5), 684-702 (2003)

172. M-A Khalighi, N Schwartz, N Aitamer, S Bourennane, Fading reduction by aperture averaging and spatial diversity in optical wireless systems. OSA J Opt Commun Netw. 1, 580-593 (2009)

173. A García-Zambrana, C Castillo-Vázquez, B Castillo-Vázquez, Outage performance of MIMO FSO links over strong turbulence and misalignment fading channels. OSA Opt Express. 19, 13480-13496 (2011)

174. CC Davis, II Smolyaninov, The effect of atmospheric turbulence on bit-errorrate in an on-off-keyed optical wireless system. Proc SPIE Free-Space Laser Commun Laser Imag. 4489, 126-137 (2002)

175. S Trisno, II Smolyaninov, SD Milner, CC Davis, Delayed diversity for fade resistance in optical wireless communication system through simulated turbulence. Proc SPIE Opt Transmission Syst Equip WDM Netw III. 5596, 385-393 (2005). Philadelphia, Pennsylvania

176. H Henniger, A Gonzalez, Transmission scheme and error protection for simplex long-distance atmospheric FSO systems. Special Issue Mediterranean J Electron Commun Hybrid RF Opt Wirel Commun. 2(3), 118-126 (2006)

177. X Zhu, JM Kahn, Performance bounds for coded free-space optical communications through atmospheric turbulence channels. IEEE Trans Commun. 51, 1233-1239 (2003)

178. IB Djordjevic, LDPC-coded MIMO optical communication over the atmospheric turbulence channel using Q-ary pulse-position modulation. OSA Opt Express. 15, 10026-10032 (2007)

179. JA Greco, Design of the high-speed framing, FEC, and interleaving hardware used in a $5.4 \mathrm{~km}$ free-space optical communication experiment. SPIE Proc Free-space Laser Communications IX. 7464 (2009)

180. H Henniger, F David, D Giggenbach, C Rapp, Evaluation of FEC for the atmospheric optical IM/DD channel. SPIE Proc Free-Space Laser Communication Technologies XV. 4975 (2003)

181. JA Anguita, MA Neifeld, B Hildner, B Vasic, Rateless coding on experimental temporally correlated FSO channels. IEEE/OSA J Lightw Technol. 28(7), 990-1002 (2010)

182. DL Fried, Limiting resolution looking down through atmosphere. JOSA. 56 1380-1384 (1966)

183. DL Fried, Aperture averaging of scintillation. JOSA. 57, 169-172 (1967)

184. DL Fried, JB Seidman, Laser-beam scintillation in the atmosphere. JOSA. 57, 181-185 (1967)

185. DP Greenwood, DL Fried, Power spectra requirements for wave-frontcompensative systems. JOSA. 66, 193-206 (1976)

186. DL Fried, Anisoplanatism in adaptive optics. JOSA. 72, 52-61 (1982)

187. JD Barchers, DL Fried, Optimal control of laser beams for propagation through a turbulent medium. JOSA. A, 1779-1793 (2002)

188. Z Sodnik, B Furch, H Lutz, Optical intersatellite communication. IEEE J Sel Topics Quantum Electron. 16(5), 1051-1057 (2010)

189. T Tolker-Nielsen, GT Oppenhaeuser, In orbit test result of an operational optical intersatellite link between ARTEMIS and SPOT4, SILEX. Proc SPIE. 6635(59430) (2002)

190. T Jono, Y Takayama, N Kura, K Ohinata, Y Koyama, K Shiratama, Z Sodnik, B Demelenne, A Bird, K Arai, OICETS on-orbit laser communication experiments. Proc SPIE. 6105(610503), 1-11 (2006) 
191. Y Fujiwara, T Jono, T Yamawaki, K Arai, M Toyoshima, H Kunimori, Z Sodnik, A Bird, B Demelenne, Optical inter-orbit communications engineering test satellite (OICETS). Acta Astronautica. 61(1-6), 163-175 (2007)

192. B Smutny, H Kampfner, G Muhlnikel, U Sterr, B Wandernoth, F Heine, U Hildebrand, D Dallmann, M Reinhardt, A Freier, R Lange, K Bohmer, T Feldhaus, J Muller, A Weichert, S Seel, R Meyer, R Czichy, $5.6 \mathrm{~Gb} / \mathrm{s}$ optical intersatellite communication link. Proc SPIE. 7199(719906) (2009)

193. KE Wilson, JR Lesh, TY Yan, GOPEX: a laser uplink to the Galileo spacecraft on its way to Jupiter. Proc SPIE. 1866, 138-146 (1993)

194. KE Wilson, JR Lesh, K Araki, Y Arimoto, Overview of the ground-to-orbit lasercom demonstration (GOLD). Proc SPIE. 2990, 23 (1997)

195. M Toyoshima, S Yamakawa, T Yamawaki, K Arai, M Reyes, A Alonso, Z Sodnik, B Demelenne, Ground-to-satellite optical link tests between Japanese laser communications terminal and European geostationary satellite ARTEMIS. Proc SPIE. 5338, 1-15 (2004)

196. J Horwath, N Perlot, M Knapek, F Moll, Experimental verification of optical backhaul links for high-altitude platform networks: atmospheric turbulence and downlink availability. Int J Satell Commun Netw. 25(5), 501-528 (2007)

197. N Perlot, M Knapek, D Giggenbach, J Horwath, M Brechtelsbauer, Y Takayama, T Jono, Results of the optical downlink experiment KIODO from OICETS satellite to optical ground station oberpfaffenhofen (OGS-OP). Proc SPIE. 6457(645704) (2007)

198. J Horwath, C Fuchs, Aircraft to ground unidirectional laser-comm terminal for high resolution sensors. Proc SPIE. 7199(719909) (2009)

199. G Baister, K Kudielka, T Dreischer, M Tüchler, Results from the DOLCE (deep space optical link communications experiment) Project. Proc SPIE. 7199(71990B) (2009)

200. IL Pe'er, N Naftali, A Yogev, High power, solar pumped, Nd:YAG, laser amplifier for free space laser communication. Proc SPIE. 3139, 194-204 (1997)

201. E Rochat, R Dändliker, K Haroud, RH Czichy, U Roth, D Costantini, R Holzner, Fiber amplifiers for coherent space communication. IEEE J Sel Topics Quantum Electron. 7(1), 64-81 (2001)

202. A Polishuk, S Arnon, Optimization of a laser satellite communication system with an optical preamplifier. J Opt Soc Am A. 21(7), 1307-1315 (2004)

203. JW Dawson, MJ Messerly, RJ Beach, MYSEA Stappaerts, AK Sridharan, PH Pax, JE Heebner, CW Siders, CPJ Barty, Analysis of the scalability of diffraction-limited fiber lasers and amplifiers to high average power. OSA Opt Express. 16(17), 13240-13266 (2008)

204. GM Djuknic, J Freidenfelds, Y Okunev, Establishing wireless communications services via high-altitude aeronautical platforms: a concept whose time has come? IEEE Commun. Mag. 35(9), 128-135 (1997)

205. M Knapek, J Horwath, F Moll, B Epple, N Courville, H Bischl, D Giggenbach, Optical high-capacity satellite downlinks via high-altitude platform relays. Proc SPIE. 6709(67090E) (2007)

206. F Fidler, M Knapek, J Horwath, WR Leeb, Optical communications for highaltitude platforms. IEEE J Sel Topics Quantum Electron. 16(5), 1058-1070 (2010)

207. DM Boroson, A Biswas, BL Edwards, MLCD: Overview of NASA's Mars laser communications demonstration system. Proc SPIE. 5338, 16-28 (2004)

208. JA Mendenhall, LM Candell, PI Hopman, G Zogbi, DM Boroson, DO Caplan, CJ Digenis, DR Hearn, RC Shoup, Design of an optical photon counting array receiver system for deep-space communications. Proc IEEE. 95(10), 2059-2069 (2007)

209. B Moision, J Hamkins, Deep-space optical communications downlink budget: modulation and coding. IPN Prog Rep. 42-154, 1-28 (2003)

210. R Lange, B Smutny, B Wandernoth, R Czichy, D Giggenbach, 142 km, 5.625 $\mathrm{Gb} / \mathrm{s}$ free-space optical link based on homodyne BPSK modulation. Proc SPIE. 6105(61050A) (2006)

211. SS Muhammad, T Javornik, I Jelovcan, Z Ghassemlooy, E Leitgeb, Comparison of hard-decision and soft-decision channel coded M-ary PPM performance over free space optical links. Eur Trans Telecommun. 20(8), 746-757 (2008)

212. IB Djordjevic, B Vasic, MA Neifeld, Multilevel coding in free-space optical MIMO transmission with Q-ary PPM over the atmospheric turbulence channel. IEEE Photon Technol Lett. 18(14), 1491-1493 (2006)

213. M Toyoshima, Y Takayama, T Takahashi, K Suzuki, S Kimura, K Takizawa, T Kuri, W Klaus, M Toyoda, H Kunimori, T Jono, K Arai, Ground-to-satellite laser communication experiments. IEEE Aerospace Electron Syst Mag. 23(8), 10-18 (2008)
214. X Zhu, JM Kahn, Free-space optical communication through atmospheric turbulence channels. IEEE Trans Commun. 50(8), 1293-1300 (2002)

215. J Lee, WWS Chan, Diversity coherent and incoherent receivers for free-space optical communication in the presence and absence of interference. OSA J Opt Commun Netw. 1(5), 463-483 (2009)

216. CC Chen, CS Gardner, Impact of random pointing and tracking errors on the design of coherent and incoherent optical intersatellite communication links. IEEE Trans Commun. 37(3), 252-260 (1989)

217. X Liu, Optimal transmitter power of an intersatellite optical communication system with reciprocal Pareto fading. OSA Appl Opt. 49, 915-919 (2010)

218. S Arnon, NS Kopeika, D Kedar, A Zilberman, D Arbel, A Livne, M Guelman, M Orenstain, H Michalik, A Ginati, Performance limitation of laser satellite communication due to vibrations and atmospheric turbulence: down-link scenario. Int J Satell Commun Netw. 21(6), 561-573 (2003)

219. N Perlot, Atmospheric occultation of optical intersatellite links: coherence loss and related parameters. OSA Appl Opt. 48(12), 2290-2302 (2009)

220. S Arnon, S Rotman, NS Kopeika, Beam width and transmitter power adaptive to tracking system performance for free-space optical communication. OSA Appl Opt. 36(24), 6095-6101 (1997)

221. GG Ortiz, S Lee, S Monacos, M Wright, A Biswas, Design and development of a robust ATP subsystem for the altair UAV-to-ground lasercomm $2.5 \mathrm{~Gb} / \mathrm{s}$ demonstration. Proc SPIE. 4975, 103-114 (2003)

222. LB Stotts, LC Andrews, PC Cherry, JJ Foshee, PJ Kolodzy, WK Mcintire, M Northcott, RL Phillips, HA Pike, B Stadler, DW Young, Hybrid optical RF airborne communications. Proc IEEE. 97(6), 1109-1127 (2009)

223. SD Milner, CC Davis, Hybrid free space optical/RF networks for tactical operations, in Proc IEEE Milcom 1, Monterey, pp. 409-415 (2004)

224. H Izadpanah, T Elbatt, V Kukshya, F Dolezal, BK Ryu, High-availability free space optical and RF hybrid wireless networks. IEEE Wirel Commun. 10(2), 45-53 (2003)

225. J Derenick, C Thorne, S Spletzer, On the deployment of a hybrid FSO/RF mobile ad-hoc network, in Proc IEEE/RSJ Int Conf Intelligent Robots and Syst, Edmonton, pp. 3990-3996 (August 2005)

226. H Tapse, DK Borah, Hybrid Optical/RF channels: characterization and performance study using low density parity check codes. IEEE Trans Commun. 57, 3288-3297 (2009)

227. V Kukshya, TS Rappaport, H Izadpanah, G Tangonan, RA Guerrero, JK Mendoza, B Lee, Free-space optics and high-speed RF for next generation networks-propagation measurements, in Proc IEEE VTC-Fall 1, Vancouver, pp. 616-620 (2002)

228. DK Borah, DG Voelz, Pointing error effects on free space optical communication links in the presence of atmospheric turbulence. IEEE/OSA J Lightwave Technol. 27, 3965-3973 (2009)

229. A Akbulut, HG IIk, F Ari, Design, availability and reliability analysis on an experimental outdoor FSO/RF communication system. Proc Transparent Opt Netw. 1, 403-406 (2005)

230. W Zhang, S Hranilovic, C Shi, Soft-switching hybrid FSO/RF links using short-length raptor codes: design and implementation. IEEE J Sel Areas Commun. 27, 1698-1708 (2009)

231. A Abdul Hussein, A Oka, T Nguyen, L Lampe, Rateless coding for hybrid free-space optical and radio-frequency communication. IEEE Trans Wirel Commun. 9, 907-913 (2010)

232. S Vangala, H Pishro-Nik, A highly reliable FSO/RF communication system using efficient codes, in Proc IEEE Globecom, Washington, D.C., pp. 2232-2236 (2007)

233. R Luna, DK Borah, R Jonnalagadda, D Voelz, Experimental demonstration of a hybrid link for mitigating atmospheric turbulence effects in free space optical communication. IEEE Photon Technol Lett. 21, 1196-1198 (2009)

234. B He, R Schober, Bit-interleaved coded modulation for hybrid RF/FSO systems. IEEE Trans Commun. 57, 3753-3763 (2009)

235. H Tapse, DK Borah, J Perez-Ramirez, Hybrid optical/RF channel performance analysis for turbo codes. IEEE Trans Commun. 59, 1389-1399 (2011)

doi:10.1186/1687-1499-2012-91

Cite this article as: Borah et al:: A review of communication-oriented optical wireless systems. EURASIP Journal on Wireless Communications and Networking 2012 2012:91. 